\title{
Conformal Invariants from Nodal Sets. I. Negative Eigenvalues and Curvature Prescription
}

\section{Yaiza Canzani $^{1}$, Rod Gover ${ }^{2,3}$, Dmitry Jakobson ${ }^{1}$, and Raphaël Ponge ${ }^{4}$, With an Appendix by Rod Gover and Andrea Malchiodi*}

${ }^{1}$ Department of Mathematics and Statistics, McGill University, Montreal, Quebec, Canada, ${ }^{2}$ Department of Mathematics, University of Auckland, Auckland, New Zealand, ${ }^{3}$ Mathematical Sciences Institute, Australian National University, Canberra, Australia, and ${ }^{4}$ Department of Mathematical Sciences, Seoul National University, Seoul, Republic of Korea

Correspondence to be sent to: e-mail: jakobson@math.mcgill.ca

In this paper, we study conformal invariants that arise from nodal sets and negative eigenvalues of conformally covariant operators; more specifically, the Graham, Jenne, Mason, and Sparling (GJMS) operators, which include the Yamabe and Paneitz operators. We give several applications to curvature prescription problems. We establish a version in conformal geometry of Courant's Nodal Domain Theorem. We also show that on any manifold of dimension $n \geq 3$, there exist many metrics for which our invariants are nontrivial. We prove that the Yamabe operator can have an arbitrarily large number of negative eigenvalues on any manifold of dimension $n \geq 3$. We obtain similar results for some higher order GJMS operators on some Einstein and Heisenberg manifolds. We describe the invariants arising from the Yamabe and Paneitz operators associated to left-invariant metrics on Heisenberg manifolds. Finally, in Appendix, the second named author and Andrea Malchiodi study the $Q$-curvature prescription problems for noncritical $Q$-curvatures.

\footnotetext{
*AM: SISSA, Trieste, Italy. Email: malchiod@sissa.it.

Received August 15, 2012; Revised November 10, 2012; Accepted December 18, 2012

(C) The Author(s) 2013. Published by Oxford University Press. All rights reserved. For permissions, please e-mail: journals.permissions@oup.com.
} 


\section{Introduction}

Nodal sets (i.e., zero loci) of eigenfunctions were first considered in the eighteenth century by Ernst Chladni in his 1787 paper, Entdeckungen über die Theorie des Klanges, on vibrating plates. More recently, some important results about nodal sets were obtained by Courant [28], Pleijel [76], Cheng and Yau [25], Donnelly and Fefferman [31], among others. For high-energy eigenfunctions of the Laplacian geometry and topology of nodal sets and nodal domains (i.e., connected components of complements of nodal sets) have also been studied in quantum chaos, in particular, in connection to random wave theory (see, e.g., [7, 70, 79]).

Conformally invariant operators with leading term a power of the Laplacian $\Delta_{g}$ have been central in mathematics and physics for over 100 years. The earliest known of these is the conformally invariant wave operator which was first constructed for the study of massless fields on curved spacetime (see, e.g., Dirac [32]). Its Riemannian signature elliptic variant, usually called the Yamabe operator, controls the transformation of the Ricci scalar curvature under conformal rescaling and so plays a critical role in the Yamabe problem on compact Riemannian manifolds. A conformal operator with principal part $\Delta_{g}^{2}$ is due to Paneitz [74], and sixth-order analogs were constructed by Branson [9] and Wünsch [80].

Two decades ago, Graham, Jenne, Mason, and Sparling (GJMS) solved a major existence problem in [50], where they used the ambient metric of Fefferman and Graham $[36,38]$ to show the existence of conformally invariant differential operators $P_{k, g}$ (to be referred to as the GJMS operators) with principal part $\Delta_{g}^{k}$. In odd dimensions, $k$ is any positive integer, while in dimension $n$ even, $k$ is a positive integer no more than $\frac{n}{2}$. The $k=1$ and $k=2$ cases recover the Yamabe and Paneitz operators, respectively. Furthermore, the GJMS operators are intimately related to the $Q_{k}$-curvatures $Q_{k, g}$ which were identified by Branson [10, 11] (see also Section 2); the $Q_{\frac{n}{2}}, g$-curvature is also known as Branson's $Q$-curvature.

The aim of this article is to study conformal invariants arising from nodal sets and negative eigenvalues of GJMS operators. In particular, we give some applications to curvature prescription problems. Various authors have considered spectral theoretic functions associated to conformally covariant operators, for example, Parker and Rosenberg [75], Osgood et al. [73], Branson and Ørsted [15], Branson et al. [12], Chang and Yang [23], and Okikiolu [72]. However, to our knowledge this is the first time that nodal sets have been considered generally in the setting of conformal geometry.

A first observation is that nodal sets and nodal domains of any null-eigenfunction of a GJMS operator are conformal invariants (Proposition 3.1). 
In case of the critical GJMS operator $P_{\frac{n}{2}}, g$, it can be further shown this feature is actually true for any level set (Proposition 3.3). Note that these results actually hold for more general conformally invariant operators, including the fractional conformal powers of the Laplacian (see Remark 3.5).

Here, we also look at the negative eigenvalues of the GJMS operators. In particular, we show that the number of negative eigenvalues of the GJMS operator is a conformal invariant (Theorem 4.2). It was shown by Kazdan and Warner [63] that the sign of the first eigenvalue of the Yamabe operator is a conformal invariant. We prove that this result actually holds for all GJMS operators (Theorem 4.3). Once again these results hold for general conformally invariant operators (see Remark 4.5) acting between the same spaces.

A natural question is whether, for a given operator, the number of negative eigenvalues can become arbitrarily large as the conformal class varies. We prove that this indeed the case for the Yamabe operator on any connected manifold (Theorem 4.6). The proof relies on a deep existence result of Lokhamp [66]. We give a more explicit proof in case of products with hyperbolic surfaces (see Proposition 4.7). Furthermore, on the product of a hyperbolic manifold with a hyperbolic surface, we construct hyperbolic metrics for which various higher order GJMS operators have arbitrary large numbers of negative eigenvalues (see Theorem 4.8 for the precise statement). In addition, we prove a version of Courant's nodal domain theorem in conformal geometry: if the Yamabe operator has $m$ negative eigenvalues, then its null-eigenfunctions have at most $m+1$ nodal domains (Theorem 4.10).

The problem of prescribing the curvature (Gaussian or scalar) of a given compact manifold is very classical and is known as the Kazdan-Warner problem (see [4, 8, 62] and the references therein). The extension of this question to Branson's $Q$-curvature has proved to be an important problem for the development of mathematical ideas (see, e.g., $[6,17,22,23,29,30,69,71])$.

We look at some constraints on curvature prescription in terms of nodal sets. A main result is Theorem 5.2 which states that if $u$ is a null-eigenfunction for the Yamabe operator $P_{1, g}$ and $\Omega$ is a nodal domain of $u$, then

$$
\int_{\Omega}|u| P_{1, g}(v) \mathrm{d} v_{g}=-\int_{\partial \Omega} v\left\|^{g} \nabla u\right\|_{g} \mathrm{~d} \sigma_{g} \quad \forall v \in C^{\infty}(M, \mathbb{R}) .
$$

Another main result is Theorem 5.5 which asserts that if a function $f$ is the scalar curvature of some metric in the conformal class of $g$, then there is a smooth function $\omega>0$ such that, for any null-eigenfunction $u$ of the Yamabe operator and any nodal 
domain $\Omega$ of $u$,

$$
\int_{\Omega} f|u| \omega \mathrm{d} v_{g}<0
$$

As a corollary, we see that if $R_{\hat{g}}$ is the scalar curvature of some metric in the conformal class of $g$, then $R_{\hat{g}}$ cannot be positive everywhere on $\Omega$.

We illustrate our results on nodal sets and negative eigenvalues in the case of the Yamabe and Paneitz operators associated to left-invariant metrics on a Heisenberg manifold $\Gamma \backslash \mathbb{H}_{d}$, obtained as the quotient of the $(2 d+1)$-dimensional Heisenberg $\mathbb{H}_{d}$ group by some lattice $\Gamma$. Using the representation theory of the Heisenberg group, we are able to give spectral resolutions for the Yamabe and Paneitz operators (see Propositions 6.3 and 6.9). Interestingly enough, some of the eigenfunctions involve theta-functions. As a result, this enables us to explicitly describe their nodal sets (see Proposition 6.6). Furthermore, we can give lower bounds for the number of negative eigenvalues of the Yamabe and Paneitz operators, which shows that these operators can have an arbitrarily large number of negative eigenvalues; the bounds involve the volume of $\Gamma \backslash \mathbb{H}_{d}$ (see Propositions 6.5 and 6.10).

For Branson's $Q$-curvature, it was shown by Malchiodi [68] (when $\int Q=0$ ) and Gover [45] (for the general case) that if the kernel of the critical GJMS operator contains nonconstant functions, then there is an infinite-dimensional space of functions that cannot be the $Q$-curvature of any metric in the conformal class.

In Appendix, by the second named author and Andrea Malchiodi, it is shown that, surprisingly, similar results are available for the noncritical $Q$-curvatures; these are the curvature quantities $Q_{k, g}, k \neq \frac{n}{2}$. The main result is Theorem A.2 which proves that for $0 \neq u \in \operatorname{ker} P_{k, g}$ any function $s_{u}$ on $M$, with the same strict sign as $u$, cannot be $Q_{k, \hat{g}}$ for any metric $\hat{g}$ in the conformal class. In particular, this potentially obstructs achieving constant $Q_{k}$-curvature (see Theorem A.5). Theorem A.2 is also used to identify a space $\mathcal{I}$ of functions, determined by the conformal structure (and, in general, properly contained in $C^{\infty}(M, \mathbb{R})$ ), which contains the range of $Q_{k, g}$, as $g$ ranges over the conformal class (see Theorem A.4 for the precise statement).

Various open problems and conjectures are gathered in Section 7. Further invariants will be considered in a forthcoming paper [21].

The paper is organized as follows. In Section 2, we review the main definitions and properties of the GJMS operators and $Q$-curvatures. In Section 3, we study the nodal sets of the GJMS operators. In Section 4, we study the negative eigenvalues of the GJMS operators. In Section 5, we discuss curvature prescription problems. In Section 6, we study the nodal sets and negative eigenvalues of Yamabe and Paneitz associated 
to left-invariant metrics on Heisenberg manifolds. In Section 7, we present various open problems and conjectures. Finally, Appendix by the second named author and Andrea Malchiodi deals with $Q$-curvature prescriptions for noncritical $Q$-curvatures.

The results of this paper were announced in [20].

\section{GJMS Operators and $Q$-Curvatures}

Let $M$ be a Riemannian manifold of dimension $n \geq 3$. A conformally covariant differential operator of biweight $\left(w, w^{\prime}\right)$ is a covariant differential operator $P_{g}$ such that, under any conformal change of metric $\hat{g}:=\mathrm{e}^{2 \Upsilon} g, \Upsilon \in C^{\infty}(M, \mathbb{R})$, it transforms according to the formula

$$
P_{\hat{g}}=\mathrm{e}^{-w^{\prime} \Upsilon} P_{g} \mathrm{e}^{w \Upsilon}
$$

An important example of a conformally invariant differential operator is the Yamabe operator (a.k.a. conformal Laplacian),

$$
P_{1, g}:=\Delta_{g}+\frac{n-2}{4(n-1)} R_{g}
$$

where $R_{g}$ is the scalar curvature. This is a conformally invariant operator of biweight $\left(\frac{n}{2}-1, \frac{n}{2}+1\right)$.

Another example is the Paneitz operator,

$$
P_{2, g}:=\Delta_{g}^{2}+\delta V d+\frac{n-4}{2}\left\{\frac{1}{2(n-1)} \Delta_{g} R_{g}+\frac{n}{8(n-1)^{2}} R_{g}^{2}-2|S|^{2}\right\}
$$

where $S_{i j}=\frac{1}{n-2}\left(\operatorname{Ric}_{g_{i j}}-\frac{R_{g}}{2(n-1)} g_{i j}\right)$ is the Schouten-Weyl tensor and $V$ is the tensor $V_{i j}=$ $\frac{n-2}{2(n-1)} R_{g} g_{i j}-4 S_{i j}$ acting on 1 -forms (i.e., $\left.V\left(\omega_{i} \mathrm{~d} x^{i}\right)=\left(V_{i}^{j} \omega_{j}\right) \mathrm{d} x^{i}\right)$. The Paneitz operator is a conformally invariant operator of biweight $\left(\frac{n}{2}-2, \frac{n}{2}+2\right)$.

A generalization of the Yamabe and Paneitz operators is provided by the GJMS operators. They were constructed by Graham et al. [50] by using the ambient metric of Fefferman and Graham [36, 38] (see also [47, 51, 60] for formulas for, and features of, the GJMS operators).

Proposition 2.1 ([50]). For $k=1, \ldots, \frac{n}{2}$ when $n$ is even, and for all nonnegative integers $k$ when $n$ is odd, there is a conformally invariant operator $P_{k}=P_{k, g}$ of biweight $\left(\frac{n}{2}-k\right.$, $\left.\frac{n}{2}+k\right)$ such that

$$
P_{k, g}=\Delta_{g}^{(k)}+\text { lower order terms. }
$$


When $n$ is even the ambient metric is obstructed at finite order by FeffermanGraham's obstruction tensor (see [36, 38]). This is a conformally invariant tensor which in dimension 4 agrees with the Bach tensor. As a result, the ambient metric construction of the GJMS operators $P_{k}$ breaks down for $k>\frac{n}{2}$, when $n$ is even. In fact, as proved by Graham [48] in dimension 4 for $k=3$ and by Gover and Hirachi [46] in general, there do not exist conformally invariant operators with same leading part as $\Delta_{g}^{k}$ for $k>\frac{n}{2}$ when $n$ is even. For this reason, the operator $P_{\frac{n}{2}, g}$ is sometimes called the critical GJMS operator. Note that for $P_{\frac{n}{2}}$ the transformation law becomes

$$
P_{\frac{n}{2}, \mathrm{e}^{2 \Upsilon} g}=\mathrm{e}^{-n \Upsilon} P_{\frac{n}{2}, g} \quad \forall \Upsilon \in C^{\infty}(M, \mathbb{R}) .
$$

When $n$ is even and the metric $g$ is conformally Einstein, the Fefferman-Graham obstruction tensor vanishes, and a canonical ambient metric exists all orders (in fact, it exists on a collar), see [38] and references therein. It is also the case that on an evendimensional conformally Einstein manifold the GJMS operator family may be extended to all (even) orders in a canonical way [44]. Furthermore, when $g$ is actually Einstein, say $\operatorname{Ric}_{g}=\lambda(n-1) g$ for some $\lambda \in \mathbb{R}$, it was shown by Graham [38,49] and Gover [44] (see also Guillarmou and Naud [54] for constant sectional curvature spaces) that

$$
P_{k, g}=\prod_{1 \leq j \leq k}\left(\Delta_{g}+\frac{\lambda}{4}(n+2 j-2)(n-2 j)\right) .
$$

The GJMS operators $P_{k, g}$ are formally self-adjoint (see [37, 51]). Moreover, they are intimately related to the $Q_{k}$-curvatures identified by Branson $[10,11]$. For $k=$ $1, \ldots, \frac{n}{2}-1$ when $n$ is even and for $k \in \mathbb{N}_{0}$ when $n$ is odd, the $Q_{k}$-curvature is defined by

$$
Q_{k}=Q_{k, g}:=\frac{2}{n-2 k} P_{k, g}(1)
$$

When $n$ is even, the $Q_{\frac{n}{2}}$-curvature (a.k.a. Branson's $Q$-curvature) is defined by analytic continuation arguments (see [9, 16]; see also [37, 51]).

For instance, it follows from (2.2)-(2.3) that

$$
Q_{1, g}=\frac{1}{2(n-1)} R_{g} \quad \text { and } \quad Q_{2}=\frac{1}{2(n-1)} \Delta_{g} R_{g}+\frac{n}{8(n-1)^{2}} R_{g}^{2}-2\left|S_{2, g}\right|^{2} .
$$

As explained in [14],

$$
P_{k, g}=\delta S_{k, g} d+\frac{n-2 k}{2} Q_{k, g}
$$

where $S_{k, g}$ is an operator acting on 1 -forms. In particular, we see that the critical GJMS operator $P_{\frac{n}{2}}$ kills the constant functions. It follows from (2.7) that, when $k \neq \frac{n}{2}$, under a 
conformal change of metric $\hat{g}:=\mathrm{e}^{2 \Upsilon} g, \Upsilon \in C^{\infty}(M, \mathbb{R})$,

$$
O_{k, \hat{g}}=\mathrm{e}^{-2 k \Upsilon} Q_{k, g}+\frac{2}{n-2 k} \mathrm{e}^{-\Upsilon\left(\frac{n}{2}+k\right)} \delta S_{k, g} d \mathrm{e}^{\Upsilon\left(\frac{n}{2}-k\right)} .
$$

When $n$ is even, for $k=\frac{n}{2}$, we have

$$
Q_{\frac{n}{2}, \hat{g}}=\mathrm{e}^{-n \Upsilon} Q_{\frac{n}{2}, g}+\mathrm{e}^{-n \Upsilon} P_{\frac{n}{2}, g}(\Upsilon)
$$

Finally, let us mention that there is a rather general theory for the existence of linear conformally invariant differential operators due to Eastwood and Slovák [33]. Further conformally invariant differential objects were also constructed by, for example, Alexakis [1, 2] and Juhl [60].

\section{Nodal Sets of GJMS Operators}

In this section, we shall look at the conformal invariance of nodal sets (i.e., zero-loci) and nodal domains (i.e., connected components of complements of nodal sets) of eigenfunctions of the GJMS operators.

Throughout this section, we let $\left(M^{n}, g\right)$ be a Riemannian manifold ( $\left.n \geq 3\right)$. In addition, we let $k \in \mathbb{N}_{0}$ and further assume $k \leq \frac{n}{2}$ when $n$ is even.

It is convenient to look at conformally covariant scalar operators as linear operators between spaces of conformal densities. Throughout the sequel, we shall regard a conformal density of weight $w, w \in \mathbb{R}$, as a family $\left(u_{\hat{g}}\right)_{\hat{g} \in[g]} \subset C^{\infty}(M)$ parameterized by the conformal class $[g]$ in such way that

$$
u_{\mathrm{e}^{2 \Upsilon} g}(x)=\mathrm{e}^{-w \Upsilon(x)} u_{g}(x) \quad \forall \Upsilon \in C^{\infty}(M, \mathbb{R})
$$

We shall denote by $\mathcal{E}[w]$ the space of conformal densities of weight $w$.

The space $\mathcal{E}[w]$ can be realized as the space of smooth functions of a line bundle over $M$ as follows (see also [75]). Denote by $\mathrm{CO}(n)$, the conformal group of $\mathbb{R}^{n}$, that is, the subgroup of $\mathrm{GL}_{n}(\mathbb{R})$ consisting of positive scalar multiples of orthogonal matrices. The datum of the conformal class $[g$ ] gives rise to a reduction of the structure group of $M$ to the conformal group $\mathrm{CO}(n)$. Denote by $E[w]$, the line bundle over $M$ associated to the representation $\rho_{w}: \mathrm{CO}(n) \rightarrow \mathbb{R}_{*}^{+}$given by

$$
\rho_{w}(A)=|\operatorname{det} A|^{\frac{w}{n}} \quad \forall A \in \operatorname{CO}(n) .
$$


Any metric $\hat{g}=\mathrm{e}^{2 \Upsilon} g, \Upsilon \in C^{\infty}(M)$, in the conformal class [ $g$ ] defines a global trivialization $\tau_{\hat{g}}: E[w] \rightarrow M \times \mathbb{R}$ with transition map,

$$
\tau_{\hat{g}} \circ \tau_{g}^{-1}(x)=\mathrm{e}^{w \Upsilon(x)} \quad \forall x \in M .
$$

This gives rise to a one-to-one correspondence between smooth sections of $E[w]$ and conformal densities. Namely, to any $u \in C^{\infty}(M, E[w])$ corresponds a unique conformal density $\left(u_{\hat{g}}\right)_{\hat{g} \in[g]}$ in $\mathcal{E}[w]$ such that, for any metric $\hat{g} \in[g]$,

$$
\tau_{\hat{g}} \circ u(x)=\left(x, u_{\hat{g}}(x)\right) \quad \forall x \in M .
$$

The property that the GJMS operator $P_{k, g}$ is conformally invariant of biweight $\left(\frac{n}{2}-k, \frac{n}{2}+k\right)$ exactly means it gives rise to a linear operator,

$$
P_{k}: \mathcal{E}\left[-\frac{n}{2}+k\right] \rightarrow \mathcal{E}\left[-\frac{n}{2}-k\right],
$$

such that, for all $u=\left(u_{\hat{g}}\right)_{\hat{g} \in[g]}$ in $\mathcal{E}\left[-\frac{n}{2}+k\right]$,

$$
\left(P_{k} u\right)_{\hat{g}}(x)=\left(P_{k, \hat{g}} u_{\hat{g}}\right)(x) \quad \forall \hat{g} \in[g] \forall x \in M .
$$

In particular, this enables us to regard the nullspace of $P_{k, g}$ as a space of conformal densities. Clearly, the dimension of ker $P_{k, g}$ is an invariant of the conformal class $[g]$.

We observe that if $u=\left(u_{\hat{g}}\right)_{\hat{g} \in[g]}$ is a conformal density of weight $w$, then the nodal set the zero locus $u_{\hat{g}}^{-1}(0)$ is independent of the metric $\hat{g}$, and hence is an invariant of the conformal class $[g]$. Applying this observation to null-eigenvectors of $P_{k}$, we then obtain the following proposition.

Proposition 3.1. Let $k \in \mathbb{N}$ and further assume $k \leq \frac{n}{2}$ if $n$ is even.

(1) If $\operatorname{dim} \operatorname{ker} P_{k, g} \geq 1$, then the nodal sets and nodal domains of any nonzero null-eigenvector of $P_{k, g}$ give rise to invariants of the conformal class $[g]$.

(2) If $\operatorname{dim} \operatorname{ker} P_{k, g} \geq 2$, then (nonempty) intersections of nodal sets of nulleigenvectors of $P_{k, g}$ and their complements are invariants of the conformal class $[g]$.

Remark 3.2. A connected component $X$ of an intersection of $p$ nodal sets should generically be a co-dimension $p$ submanifold of $M$, and in the case it is, the corresponding homology class in $H_{n-p}(M)$ would be a conformal invariant. Further interesting conformal invariants should arise from considering the topology of $M \backslash X$. For example, 
if $\operatorname{dim} M=3$ and $\operatorname{dim} \operatorname{ker} P_{k}=2$, and $u_{1}, u_{2} \in \operatorname{ker} P_{k}$, then $\mathcal{N}\left(u_{1}\right) \cap \mathcal{N}\left(u_{2}\right)$ should define a "generalized link" in $M$, and all topological invariants of that set and its complement in $M$ would be conformal invariants. Related invariants are considered in [26, 27].

When $k=\frac{n}{2}$ ( $n$ even) the nullspace of $P_{\frac{n}{2}}$ is contained in the space $\mathcal{E}[0]$ of conformal densities of weight 0 and it always contains constant functions (seen as conformal densities of weight zero, that is, a constant family of constant functions).

Observe also that if $u=\left(u_{\hat{g}}\right)_{\hat{g} \in[g]}$ is a conformal density of weight 0 , then, in addition to the zero-locus, all the level sets $\left\{x \in M ; u_{g}(x)=\lambda\right\}, \lambda \in \mathbb{C}$, are independent of the representative metric $g$. Therefore, for the critical GJMS operator, we obtain the following proposition.

Proposition 3.3. Assume $n$ is even. If $\operatorname{dim} \operatorname{ker} P_{\frac{n}{2}} \geq 2$, then the level sets of any nonconstant null-eigenvector of $P_{\frac{n}{2}}$ are invariants of the conformal class $[g]$.

Next, we mention the following result.

Proposition 3.4. Assume $M$ compact and $k<\frac{n}{2}$. Let $u_{g} \in \operatorname{ker} P_{k, g}$ and let us regard $u$ as a conformal density of weight $-\frac{n}{2}+k$. Then the integral $\int_{M}\left|u_{g}(x)\right|^{\frac{2 n}{n-2 k}} \mathrm{~d} v_{g}(x)$ is an invariant of the conformal class $[g]$.

Proof. Let $\hat{g}=\mathrm{e}^{2 \Upsilon} g, \Upsilon \in C^{\infty}(M, \mathbb{R})$ be a metric in the conformal class [ $g$ ]. Then

$$
\int_{M}\left|u_{\hat{g}}(x)\right|^{\frac{2 n}{n-2 k}} \mathrm{~d} v_{\hat{g}}(x)=\int_{M}\left|\mathrm{e}^{\frac{2 k-n}{2} \Upsilon(x)} u_{g}(x)\right|^{\frac{2 n}{n-2 k}} \mathrm{e}^{n \gamma(x)} \mathrm{d} v_{g}(x)=\int_{M}\left|u_{g}(x)\right|^{\frac{2 n}{n-2 k}} \mathrm{~d} v_{g}(x) .
$$

This proves the result.

Remark 3.5. Although stated for the GJMS operators, the results of this section actually hold for any conformally covariant operator that yields an endomorphism on some function space. They even hold for conformally invariant pseudodifferential operators, including the conformal fractional powers of the Laplacian $P_{s, g}, s>0$, which extend the GJMS construction to noninteger orders (see [51,55]). More precisely, Proposition 3.1 holds verbatim for any such conformally invariant operator, and Proposition 3.3 (respectively, Proposition 3.4) holds verbatim for any such conformally invariant operator of biweight $\left(w, w^{\prime}\right)$ with $w=0$ (respectively, $w=\frac{n}{2}-k$ with $k \in\left(0, \frac{n}{2}\right)$ ). 


\section{Negative Eigenvalues of GJMS Operators}

In this section, we look at the negative eigenvalues of the GJMS operators. Throughout this section $M^{n}$ is a compact manifold ( $n \geq 3$ ) and we let $k \in \mathbb{N}$ (and further assume $k \leq \frac{n}{2}$ when $n$ is even).

Let $\mathcal{G}$ be the set of Riemannian metrics on $M$ equipped with its standard Fréchetspace $C^{\infty}$-topology. As mentioned in Section 2, given any metric $g$ on $M$, the GJMS operator $P_{k, g}$ is self-adjoint with respect to the inner product defined by $g$. Moreover, as it has same leading part as $\Delta_{g}^{k}$ it has a positive principal symbol. Therefore, it spectrum consists of a sequence of real eigenvalues converging to $\infty$. We thus can order the eigenvalues of $P_{k, g}$ as a nondecreasing sequence,

$$
\lambda_{1}\left(P_{k, g}\right) \leq \lambda_{2}\left(P_{k, g}\right) \leq \cdots,
$$

where each eigenvalue is repeated according to multiplicity. Note that by the $\min -\max$ principle,

$$
\lambda_{j}\left(P_{k, g}\right)=\inf _{\substack{E \subset C^{\infty}(M) \\ \operatorname{dim} E=j \| u \in E}} \sup _{\substack{u \in E \\\|u\|}}\left\langle P_{k, g} u, u\right\rangle .
$$

Lemma 4.1 ([64, Theorem 2]). For every $j \in \mathbb{N}$, the function $g \rightarrow \lambda_{j}\left(P_{k, g}\right)$ is continuous on $\mathcal{G}$.

For a metric $g \in \mathcal{G}$, we define

$$
v_{k}(g):=\#\left\{j \in \mathbb{N} ; \lambda_{j}\left(P_{k, g}\right)<0\right\}
$$

In addition, for any $m \in \mathbb{N}$, we set

$$
\mathcal{G}_{k, m}:=\left\{g \in \mathcal{G} ; \quad P_{k, g} \text { has at least } m \text { negative eigenvalues }\right\}
$$

Note that $\mathcal{G}_{k, m}=\left\{g \in \mathcal{G} ; v_{k}(g) \geq m\right\}=\left\{g \in \mathcal{G} ; \lambda_{m}\left(P_{k, g}\right)<0\right\}$, so it follows from Lemma 4.1 that $\mathcal{G}_{k, m}$ is an open subset of $\mathcal{G}$.

Theorem 4.2. Let $g \in \mathcal{G}$. Then $v_{k}(g)$ is an invariant of the conformal class $[g]$.

Proof. Let $g \in \mathcal{G}$, and set $m=v_{k}(g)$ and $l=\operatorname{dim} \operatorname{ker} P_{k, g}$. Thus $\lambda_{j}\left(P_{k, g}\right)<0$ for $j \leq m$, and $\lambda_{j}\left(P_{k, g}\right)=0$ for $j=m+1, \ldots, m+l$, and $\lambda_{j}\left(P_{k, g}\right)>0$ for $j \geq m+l+1$. Let $\delta$ be a positive 
real number $<\min \left\{\left|\lambda_{m}\left(P_{k, g}\right)\right|, \lambda_{m+l+1}\left(P_{k, g}\right)\right\}$. It follows from Lemma 4.1, if a metric $\hat{g}$ in the conformal class [ $g$ ] is close enough to $g$, then $\lambda_{m}\left(P_{k, \hat{g}}\right)<-\delta$ and $\lambda_{m+l+1}\left(P_{k, \hat{g}}\right)>\delta$. That is, the only $\lambda_{j}\left(P_{k, \hat{g}}\right)$ that are contained in the interval $[-\delta, \delta]$ are $\lambda_{m+1}\left(P_{k, \hat{g}}\right), \ldots, \lambda_{m+l}\left(P_{k, \hat{g}}\right)$.

As mentioned in Section 3, the dimension of $\operatorname{ker} P_{k, g}$ is an invariant of the conformal class $[g]$, so $\operatorname{dim} \operatorname{ker} P_{k, \hat{g}}=l$, that is, there are exactly $l$ of the $\lambda_{j}\left(P_{k, \hat{g}}\right)$ that are equal to 0 . Since we know that the $l$ eigenvalues $\lambda_{m+1}\left(P_{k \hat{g}}\right), \ldots, \lambda_{m+l}\left(P_{k \hat{g}}\right)$ are the only eigenvalues that are contained in $[-\delta, \delta]$, it follows that $\lambda_{m+1}\left(P_{k, \hat{g}}\right)=\cdots=\lambda_{m+l}\left(P_{k, \hat{g}}\right)=0$. As $\lambda_{m}\left(P_{k, \hat{g}}\right)<0$, we then conclude that $\nu_{k}(\hat{g})=m$.

All this shows that the map $g \rightarrow v_{k}(g)$ is locally constant when restricted to the conformal class $[g]$. As $[g]$ is a connected subset of $\mathcal{G}$ (since this is the range of $C^{\infty}(M, \mathbb{R})$ under $\Upsilon \rightarrow \mathrm{e}^{2 \Upsilon} g$ ), we deduce that $v_{k}(g)$ is actually constant along the conformal class $[g]$. This proves the theorem.

It follows from Theorem 4.2 that the number of negative eigenvalues of each GJMS operator defines a partition of the set of conformal classes.

A result of Kazdan-Warner [63, Theorem 3.2] asserts that the sign of the first eigenvalue $\lambda_{1}\left(P_{1, g}\right)$ is an invariant of the conformal class [ $g$ ]. Note that:

(i) $\lambda_{1}\left(P_{k, g}\right)<0$ if and only if $v_{k}(g) \geq 1$.

(ii) $\lambda_{1}\left(P_{k, g}\right)=0$ if and only if $\nu_{k}(g)=0$ and $\operatorname{dim} \operatorname{ker} P_{k, g} \geq 1$.

(iii) $\lambda_{1}\left(P_{k, g}\right)>0$ if and only if $\operatorname{dim} \operatorname{ker} P_{k, g}=v_{k}(g)=0$.

Therefore, as an immediate consequence of the conformal invariance of dim ker $P_{k, g}$ and $v_{k}(g)$, we obtain the following extension of Kazdan-Warner's result.

Theorem 4.3. The sign of the first eigenvalue $\lambda_{1}\left(P_{k, g}\right)$ is an invariant of the conformal class $[g]$.

Remark 4.4. Let $g_{0}$ be a metric of constant scalar curvature in the conformal class. As the nullspace of the Laplacian consists of constant functions, $\lambda_{1}\left(P_{1, g_{0}}\right)=\frac{n-2}{4(n-1)} R_{g_{0}}$. Therefore, the sign of $\lambda_{1}\left(P_{1, g}\right)$ agrees with that of the constant scalar curvature $R_{g_{0}}$. We also see that $\lambda_{1}\left(P_{k, g}\right)=0$ if and only if $R_{g_{0}}=0$. Furthermore, in that case ker $P_{1, g_{0}}$ consists of constant functions and ker $P_{1, g}$ is spanned by a single positive function.

Remark 4.5. Both Theorems 4.2 and 4.3 hold verbatim for any conformally invariant pseudodifferential operator acting on functions or even on sections of a vector bundle. 
In particular, they hold for the fractional conformal powers of the Laplacian on functions.

The question that naturally arises is whether $v_{k}(g)$ can be arbitrary large as $g$ ranges over metrics on $M$. The following shows this is indeed the case when $k=1$ (i.e., $P_{k, g}$ is the Yamabe operator).

Theorem 4.6. Assume $M$ connected. Then, for every $m \in \mathbb{N}$, there is a metric $g$ on $M$ such that the Yamabe operator $P_{1, g}$ has at least $m$ negative eigenvalues counted with multiplicity.

Proof. By a result of Lohkamp [66, Theorem 2], given $\lambda>0$, there is a metric $g$ on $M$ such that:

(i) The $m$ first positive eigenvalues of the Laplacian $\Delta_{g}$ counted with multiplicity are equal to $\lambda$.

(ii) The volume of $(M, g)$ is equal to 1 .

(iii) The Ricci curvature of $g$ is $\leq-m^{2}$.

The condition (iii) implies that $R_{g} \leq-n m^{2}$. Combining this with (ii) shows that, for all $u \in C^{\infty}(M)$, we have

$$
\begin{aligned}
\left\langle P_{1, g} u, u\right\rangle & =\left\langle\Delta_{g} u, u\right\rangle+\frac{(n-2)}{4(n-1)} \int_{M} R_{g}(x)|u(x)|^{2} v_{g}(x) \\
& \leq\left\langle\Delta_{g} u, u\right\rangle-\frac{(n-2)}{4(n-1)} n m^{2}\|u\|^{2}
\end{aligned}
$$

Assume $\lambda<\frac{(n-2)}{4(n-1)} n m^{2}$ and denote by $E$ the eigenspace of $\Delta_{g}$ associated to $\lambda$. Note that $E$ is a subspace of $C^{\infty}(M)$ and has dimension $\ell \geq m$. Moreover, if $u$ a unit vector in $E$, then (4.2) shows that $\left\langle P_{1, g} u, u\right\rangle \leq \lambda-\frac{(n-2)}{4(n-1)} n m^{2}<0$. Combining this with the minmax principle (4.1), we see that $\lambda_{m}\left(P_{1, g}\right) \leq \lambda_{\ell}\left(P_{1, g}\right)<0$. Thus, $P_{1, g}$ has at least $m$ negative eigenvalues counted with multiplicity. The proof is complete.

More explicit construction of metrics with an arbitrarily large number of eigenvalues can be given in the case of a product with a hyperbolic surface.

Assume $n \geq 4$ and let $\left(N^{n-2}, g_{1}\right)$ be a compact Riemannian manifold and $\left(\Sigma, g_{2}\right)$ a hyperbolic surface of genus $\geq 2$. Given $t>0$ we equip the product $M:=N \times \Sigma$ with the product metric $g_{t}:=g_{1} \otimes 1+1 \otimes t^{-1} g_{2}$. 
Proposition 4.7. For any $m \in \mathbb{N}$, we can choose $g_{2}$ and $t$ such that the Yamabe operator $P_{1, g_{t}}$ has at least $m$ negative eigenvalues.

Proof. The scalar curvature of $g_{t}$ is $R_{g_{t}}=R_{g_{1}}-2 t$, so the Yamabe operator on $\left(M, g_{t}\right)$ is

$$
P_{1, g_{t}}=\Delta_{g_{1}} \otimes 1+t\left(1 \otimes \Delta_{g_{2}}\right)+\frac{n-2}{4(n-1)}\left(R_{g_{1}}-2 t\right)
$$

where $\Delta_{g_{1}}$ (respectively, $\Delta_{g_{2}}$ ) is the Laplacian on $N$ (respectively, $\Sigma$ ).

Let $\lambda$ be an eigenvalue of $\Delta_{g_{2}}$ and let $u$ be an associated eigenfunction. We have

$$
P_{1, g_{t}} u=t \lambda u+\frac{n-2}{4(n-1)}\left(R_{g_{1}}-2 t\right) u
$$

Set $\mu:=\frac{n-2}{4(n-1)} \sup _{x \in N} R_{g_{1}}(x)$. Then

$$
\left\langle P_{1, g_{t}} u, u\right\rangle \leq\left(t \lambda+\mu-\frac{n-2}{2(n-1)} t\right)\langle u, u\rangle=t\left(\lambda-\frac{n-2}{2(n-1)}+t^{-1} \mu\right)\langle u, u\rangle .
$$

Let $m \in \mathbb{N}$. Observe that $\frac{n-2}{2(n-1)}=\frac{1}{2}-\frac{1}{2(n-1)}>\frac{1}{4}$ since $n \geq 4$. Therefore, we may choose $t$ large enough so that $\frac{n-2}{2(n-1)}-t^{-1} \mu>\frac{1}{4}$. Then a result of Buser [19, Theorem 4] ensures us that we can choose the metric $g_{2}$ so that $\Delta_{g_{2}}$ has at least $m$ eigenvalues $\lambda<\frac{n-2}{2(n-1)}-t^{-1} \mu$. If $u$ is an eigenfunction of $u$ associated to such an eigenvalue, then (4.3) shows that $\langle P u, u\rangle<0$. Therefore, the quadratic form defined by $P_{1, g_{t}}$ is negative definite on an $m$-dimensional subspace of $C^{\infty}(M)$. Applying the min-max principle (4.1), then shows that $\lambda_{m}\left(P_{\left.1, g_{t}\right)}<0\right.$, that is, $P_{1, g_{t}}$ has at least $m$ negative eigenvalues. The proof is complete.

Next, we construct explicit examples of products of hyperbolic manifolds for which various higher order GJMS operators have an arbitrarily large number of negative eigenvalues upon varying the metric.

Let $\left(N^{n-2}, g_{1}\right)$ be a hyperbolic manifold and $\left(\Sigma^{2}, g_{2}\right)$ a hyperbolic surface of genus $\geq 2$. We equip the product manifold $M:=N \times \Sigma$ with the product metric $g:=g_{1} \otimes 1+1 \otimes g_{2}$. Note that $(M, g)$ is an Einstein manifold and Ric $=-g$.

Theorem 4.8. For every $m \in \mathbb{N}$, we can choose the (hyperbolic) metric $g_{2}$ on $\Sigma$ so that the GJMS operator $P_{k, g}$ has at least $m$ negative eigenvalues for all odd integers $k \leq \frac{n-1}{2}$. 
If we further assume that $n=4 l$ or $n=4 l+1$ for some $l \in \mathbb{N}$, then the same conclusion holds for all integers $k \geq \frac{n}{2}$.

Remark 4.9. As $(M, g)$ is Einstein, the GJMS operator $P_{k}$ family extends to a family of canonical Laplacian power type operators defined for all integers $k>\frac{n}{2}$ even when $n$ is even [44].

Proof of Theorem 4.8. As Ric $=-g$, Equation (2.5) gives

$$
P_{k, g}=\prod_{1 \leq j \leq k}\left(\Delta_{g}-\mu_{j}\right), \quad \mu_{j}:=\frac{1}{4(n-1)}(n+2 j-2)(n-2 j) .
$$

Note also that the Laplacian on $M$ is $\Delta_{g}=\Delta_{g_{1}} \otimes 1+1 \otimes \Delta_{g_{2}}$, where $\Delta_{g_{1}}$ (respectively, $\Delta_{g_{2}}$ ) is the Laplacian on $N$ (respectively, $\Sigma$ ).

Let $\lambda$ be an eigenvalue of $\Delta_{g_{2}}$ and let $u$ be an eigenfunction associated to $\lambda$. If we regard $u$ as a function on $M$, then $\Delta_{g} u=\Delta_{g_{2}} u=\lambda u$. Combining this with (4.4) we then see that $u$ is an eigenvector of $P_{k, g}$ with eigenvalue

$$
\Lambda_{k}:=\prod_{1 \leq j \leq k}\left(\lambda-\mu_{j}\right)
$$

Observe that $4(n-1) \mu_{j}=(n-1)^{2}-(2 j-1)^{2}$, so $\mu_{j}>\mu_{j+1}$ for $j \geq \frac{1}{2}$. Moreover, $\mu_{\frac{n}{2}}=0$ and $\mu_{\frac{n-1}{2}}=\frac{2 n-3}{4(n-1)}=\frac{1}{2}-\frac{1}{4(n-1)}>\frac{1}{4}$. Incidentally, $\mu_{j} \geq 0$ when $j \geq \frac{n}{2}$.

Let $m \in \mathbb{N}$. As $\mu_{\frac{n-1}{2}}>\frac{1}{4}$ and $\Sigma$ has genus $\geq 2$, appealing again to [19, Theorem 4] we can find a hyperbolic metric $g_{2}$ such that the Laplacian $\Delta_{g_{2}}$ has at least $m$ eigenvalues contained in $\left(0, \mu_{\frac{n-1}{2}}\right)$. Let $\lambda$ be such an eigenvalue and assume that $k$ is an odd integer $\leq$ $\frac{n-1}{2}$. Then $\lambda-\mu_{j} \leq \lambda-\mu_{k} \leq \lambda-\mu_{\frac{n-1}{2}}<0$ for $j=1, \ldots, k$, and so the eigenvalue $\Lambda_{k}$ in (4.5) is the product of $k$ negative numbers. As $k$ is odd, it follows that $\Lambda_{k}$ is a negative eigenvalue of $P_{k, g}$. This enables us to produce $m$ negative eigenvalues for this operator.

Assume further that $m=4 l$ or $m=4 l+1$ for some $l \in \mathbb{N}$ and suppose that $k$ is an integer $\geq \frac{n}{2}$. Then the integral part $k_{0}=\left[\frac{n-2}{2}\right]$ is an odd number and we can write

$$
\Lambda_{k}=\Lambda_{k}^{\prime} \Lambda_{k_{0}} \quad \text { with } \Lambda_{k}^{\prime}:=\prod_{k_{0}+1 \leq j \leq k}\left(\lambda-\mu_{j}\right)
$$

Note that $\Lambda_{k_{0}}<0$ since $k_{0}$ is an odd integer $\leq \frac{n-1}{2}$. Moreover, as $k_{0}+1 \geq \frac{n}{2}$, we see that $\mu_{j} \leq 0$ for all $j \geq k_{0}+1$, and hence $\Lambda_{k}^{\prime}$ is a positive number. It then follows that $\Lambda_{k}$ is 
a negative eigenvalue of $P_{k, g}$, and so this operator has $m$ negative eigenvalues for this operator. The proof is complete.

Finally, we derive a version of Courant's nodal domain theorem in conformal geometry.

Theorem 4.10. Assume that the Yamabe operator $P_{1, g}$ has $m \geq 1$ negative eigenvalues. Then any null eigenfunction of $P_{1, g}$ has at most $m+1$ nodal domains.

Proof. By Proposition 3.1, the nodal domains of $P_{1, g}$ are conformal invariants. Therefore, without any loss of generality, we may assume that the scalar curvature $R_{g}$ is constant. Then the eigenvalues of $P_{1, g}$ are obtained by adding $c=\frac{n-2}{4(n-1)} R_{g}$ to the eigenvalues of the Laplacian $\Delta_{g}$ and the corresponding eigenspaces agrees.

Let $u \in \operatorname{ker} P_{1, g}$. By assumption $P_{1, g}$ has $m$ negative eigenvalues, and so the eigenvalue $\lambda=0$ is the $j$ th eigenvalue of $P_{1, g}$ for some $j \geq m$. It then follows that $u$ is an eigenfunction of $\Delta_{g}$ for its $j$ th eigenvalue. Applying Courant's nodal domain theorem [24, 28], we then see that $u$ has at most $m+1$ nodal domains. The proof is complete.

\section{Curvature Prescription Problems}

In this section, we look at some constraints on curvature prescription. Further results are given in Appendix. The problem of prescribing the curvature (Gaussian or scalar) of a given compact manifold is very classical and is known as the Kazdan-Warner problem (see $[4,8,62]$ and the references therein). The extension of this question to Branson's $Q$-curvature is an important impetus for the development of various mathematical ideas (see, e.g., [6, 17, 22, 23, 29, 30, 69, 71]).

Throughout this section, we let $\left(M^{n}, g\right)$ be a compact Riemannian manifold $\left(n \geq 3\right.$ ). Given $k \in \mathbb{N}$ (further assuming $k \leq \frac{n}{2}$ if $n$ is even), the problem of conformally prescribing $Q_{k}$-curvature is that of determining which functions can be expressed as the $O_{k}$-curvature of some metric $\hat{g}$ in the conformal class $[g]$. In other words, we seek to characterize the range $\mathcal{R}\left(O_{k}\right)$ of the map,

$$
Q_{k}:[g] \ni \hat{g} \longrightarrow Q_{k, \hat{g}} \in C^{\infty}(M, \mathbb{R})
$$

Let us first make some elementary observations on $\int_{M} R_{g} u \mathrm{~d} v_{g}$ with $u \in \operatorname{ker} P_{1, g}$. As $Q_{1, g}=\frac{1}{2(n-1)} R_{g}$, using elementary spectral considerations and expression (A.4) in Appendix, we obtain the following proposition. 
Proposition 5.1. Assume that the scalar curvature $R_{g}$ is constant. Then

$$
\int_{M} R_{g} u \mathrm{~d} v_{g}=0 \quad \forall u \in \operatorname{ker} P_{1, g}
$$

In addition, if $\hat{g}=\mathrm{e}^{2 \Upsilon} g, \Upsilon \in C^{\infty}(M, \mathbb{R})$, is a metric in the conformal class $[g]$, then

$$
\int_{M} \mathrm{e}^{\frac{2-n}{2} \Upsilon} R_{\hat{g}} u \mathrm{~d} v_{\hat{g}}=0 \quad \forall u \in \operatorname{ker} P_{1, g}
$$

Next, we consider the scalar curvature restricted to nodal domains. The following extends to the Yamabe operator a formula of Sogge and Zelditch [78, Proposition 1] for the Laplace operator.

Theorem 5.2. Let $u \in \operatorname{ker}\left(P_{1, g}\right)$ and let $\Omega$ be a nodal domain of $u$. Then, for all $v \in C^{\infty}(M)$,

$$
\int_{\Omega}|u| P_{1, g}(v) \mathrm{d} v_{g}=-\int_{\partial \Omega} v\left\|^{g} \nabla u\right\|_{g} \mathrm{~d} \sigma_{g}
$$

where ${ }^{g} \nabla$ is the Levi-Civita connection of $g$ and $\sigma_{g}$ is the surface measure of $\partial \Omega$.

Remark 5.3. The intersection of the critical and nodal sets of $u$ has locally finite $n$-2-Hausdorff dimension ([57, 58]; see also $[24,56])$. Therefore, $\partial \Omega$ admits a normal vector almost everywhere, and hence the surface measure $d \sigma_{g}$ is well-defined.

Proof of Theorem 5.2. Note that $u$ has constant sign on $\Omega$. Let $v$ be the outward unit normal vector to the hypersurface $\partial \Omega$. Then $\partial_{\nu} u$ agrees with $-\left\|^{g} \nabla u\right\|_{g}$ (respectively, $\left\|^{g} \nabla u\right\|_{g}$ ) almost everywhere on $\partial \Omega$ in case $u$ is positive (respectively, negative) on $\Omega$. Therefore, possibly upon replacing $u$ by $-u$, we may assume that $u$ is positive on $\Omega$.

Let $v \in C^{\infty}(M)$. As $P_{1, g} u=0$ and the Yamabe operator agrees with the Laplacian $\Delta_{g}$ up to a the multiplication by a function, we have

$$
\int_{\Omega}|u| P_{1, g}(v) \mathrm{d} v_{g}=\int_{\Omega}\left(u P_{1, g} v-v P_{1, g} u\right) \mathrm{d} v_{g}=\int_{\Omega}\left(u \Delta_{g} v-v \Delta_{g} u\right) \mathrm{d} v_{g}
$$

Note that $u \Delta_{g} v-v \Delta_{g} u=u \operatorname{div}\left({ }^{g} \nabla v\right)-u \operatorname{div}\left({ }^{g} \nabla v\right)=\operatorname{div}\left(u\left({ }^{g} \nabla v\right)-v\left({ }^{g} \nabla u\right)\right)$. Therefore, applying the divergence theorem for rough domains (see [35, Section 4.5.6]), we deduce 
that the integral $\int_{\Omega}|u| P_{1, g}(v) \mathrm{d} v_{g}$ is equal to

$$
\int_{\Omega} \operatorname{div}\left(u\left({ }^{g} \nabla\right) v-v\left({ }^{g} \nabla u\right)\right) \mathrm{d} v_{g}=-\int_{\partial \Omega}\left(u \partial_{\nu} v-v \partial_{\nu} u\right) \mathrm{d} \sigma_{g}=-\int_{\partial \Omega} v\left\|^{g} \nabla u\right\|_{g} \mathrm{~d} \sigma_{g},
$$

where we have used the fact that $u=0$ and $\partial_{\nu} u=-\left\|^{g} \nabla u\right\|_{g}$ on $\partial \Omega$. The proof is complete.

Decomposing the manifold into a disjoint union of positive nodal domains, negative nodal domains and the nodal set of $u$, and applying Theorem 5.2 we obtain:

Corollary 5.4. For all $u \in \operatorname{ker} P_{1, g}$ and $v \in C^{\infty}(M)$,

$$
\int_{M}|u| P_{1, g}(v) \mathrm{d} v_{g}=-2 \int_{\mathcal{N}(u)} v\left\|^{g} \nabla u\right\|_{g} \mathrm{~d} \sigma_{g}
$$

where $\mathcal{N}(u)$ is the nodal set of $u$.

Theorem 5.5. Let $f \in C^{\infty}(M)$ be the scalar curvature of some metric in the conformal class [g]. Then, there is a positive function $\omega \in C^{\infty}(M)$, such that, for any $u \in \operatorname{ker}\left(P_{1, g}\right)$ and any nodal domain $\Omega$ of $u$,

$$
\int_{\Omega} f|u| \omega \mathrm{d} v_{g}<0
$$

Proof. By assumption $f=R_{\hat{g}}$ for some metric $\hat{g}=\mathrm{e}^{2 \Upsilon} g, \Upsilon \in C^{\infty}(M, \mathbb{R})$. Thus $P_{1, \hat{g}}(1)=$ $\frac{n-2}{4(n-1)} R_{\hat{g}}=\frac{n-2}{4(n-1)} f$. Let $u \in \operatorname{ker}\left(P_{1, g}\right)$ and let $\Omega$ be nodal domain of $u$. In addition, set $\omega=$ $\frac{n-2}{4(n-1)} \mathrm{e}^{\frac{n+2}{2} \Upsilon}$ and $\hat{u}=\mathrm{e}^{\frac{2-n}{2} \Upsilon} u$. Then

$$
\int_{\Omega} f|u| \omega \mathrm{d} v_{g}=\frac{n-2}{4(n-1)} \int_{\Omega}|\hat{u}| f \mathrm{~d} v_{\hat{g}}=\int_{\Omega}|\hat{u}| P_{1, \hat{g}}(1) \mathrm{d} v_{\hat{g}} .
$$

As the kernel of the Yamabe operator consists of conformal densities of weight $\frac{n-2}{2}$, we see that $\hat{u}$ is contained in $P_{1, \hat{g}}$ and $\Omega$ is a nodal domain for $\hat{u}$. Therefore, applying Theorem 5.2 to $\hat{u}$ and $v=1$ and using (5.2), we obtain

$$
\int_{\Omega} \omega|u| f \mathrm{~d} v_{g}=\int_{\Omega}|\hat{u}| P_{1, \hat{g}}(1) \mathrm{d} v_{\hat{g}}=-\int_{\partial \Omega}\left\|{ }^{\hat{g}} \nabla u\right\|_{\hat{g}} \mathrm{~d} \sigma_{\hat{g}} .
$$

As the intersection of the critical and nodal sets of $u$ has locally finite $n$-2-Hausdorff dimension ([57, 58]; see also [24,56]), the integral $\int_{\partial \Omega}\|\|^{\hat{g}} \nabla u \|_{\hat{g}} \mathrm{~d} \sigma_{\hat{g}}$ must be positive, and hence $\int_{\Omega} \omega|u| f \mathrm{~d} v_{g}<0$. This proves the result. 
Theorem 5.5 seems to be new. We remark that when $\operatorname{dim} \operatorname{ker}\left(P_{1, g}\right) \geq 2$ this gives infinitely many constraints on $R_{\hat{g}}$.

Corollary 5.6. Let $u \in \operatorname{ker}\left(P_{1, g}\right)$ and let $\Omega$ be nodal domain of $u$. Then, for any metric $\hat{g}$ in the conformal class $[g]$, the scalar curvature $R_{\hat{g}}$ cannot be everywhere positive on $\Omega$.

Let $u \in \operatorname{ker}\left(P_{1, g}\right)$ and let $\Omega$ be nodal domain of $u$. Given any metric $\hat{g}=\mathrm{e}^{2 \Upsilon} g, \Upsilon \in$ $C^{\infty}(M, \mathbb{R})$, in the conformal class $[g]$ we define

$$
T(u, \Omega, \hat{g}):=-\frac{4(n-1)}{n-2} \int_{\partial \Omega} \mathrm{e}^{\frac{2-n}{2} \Upsilon}\left\|^{\hat{g}} \nabla \hat{u}\right\|_{\hat{g}} \mathrm{~d} \sigma_{\hat{g}}
$$

where we have set $\hat{u}=\mathrm{e}^{\frac{2-n}{2} \Upsilon} u$.

Proposition 5.7. For all metrics $\hat{g}$ in the conformal class $[g]$,

$$
T(u, \Omega, \hat{g})=\int_{\Omega}|u| R_{g} \mathrm{~d} v_{g}
$$

Proof. Let $\hat{g}=\mathrm{e}^{2 \Upsilon} g, \Upsilon \in C^{\infty}(M, \mathbb{R})$ be a metric in the conformal class $[g]$. Set $\hat{u}=\mathrm{e}^{\frac{2-n}{2}} u$ and $v=\mathrm{e}^{\frac{2-n}{2}} \Upsilon$. As pointed out in the proof of Theorem 5.5, $\hat{u}$ lies in $\operatorname{ker} P_{1, \hat{g}}$ and $\Omega$ is a nodal domain. Applying Theorem 5.2 to $\hat{u}$ and $v$ then gives

$$
\frac{n-2}{4(n-1)} T(u, \Omega, \hat{g})=-\int_{\partial \Omega} v\left\|^{\hat{g}} \nabla \hat{u}\right\|_{\hat{g}} \mathrm{~d} \sigma_{\hat{g}}=\int_{\Omega}|\hat{u}| P_{1, \hat{g}} v \mathrm{~d} v_{\hat{g}}
$$

As $P_{1, \hat{g}} v=\mathrm{e}^{-\frac{n+2}{2} \Upsilon} P\left(\mathrm{e}^{\frac{n-2}{2} \Upsilon} \cdot \mathrm{e}^{\frac{2-n}{2} \Upsilon}\right)=\mathrm{e}^{-\frac{n+2}{2} \Upsilon} P_{1, g}(1)=\frac{n-2}{4(n-1)} \mathrm{e}^{-\frac{n+2}{2} \Upsilon} R_{g}$, we obtain

$$
T(u, \Omega, \hat{g})=\int_{\Omega}|\hat{u}| \mathrm{e}^{-\frac{n+2}{2} \Upsilon} R_{g} \mathrm{~d} v_{\hat{g}}=\int_{\Omega} \mathrm{e}^{\frac{2-n}{2} \Upsilon}|u| \mathrm{e}^{-\frac{n+2}{2} \Upsilon} R_{g} \mathrm{e}^{n \Upsilon} \mathrm{d} v_{g}=\int_{\Omega}|u| R_{g} \mathrm{~d} v_{g} .
$$

The result is proved.

Proposition 5.7 provides us with some conserved quantities for the conformal class. In particular, if $R_{g}$ is constant, then we obtain

$$
T(u, \Omega, \hat{g})=R_{g}\|u\|_{L^{1}(\Omega)} .
$$


Finally, let $k \in \mathbb{N}$ and further assume $k \leq \frac{n}{2}-1$ when $n$ is even. We look at conformal classes containing a metric for which the $Q_{k}$-curvature is zero.

Proposition 5.8. The following are equivalent:

(1) The kernel of $P_{k, g}$ contains a nowhere vanishing eigenfunction.

(2) There is a metric $\hat{g}$ in the conformal class $[g]$ such that $P_{k, \hat{g}}(1)=0$.

(3) There is a metric $\hat{g}$ in the conformal class $[g]$ such that $Q_{k, \hat{g}}$ is identically zero.

Proof. As by definition $Q_{k, \hat{g}}=\frac{2}{n-2 k} P_{k, \hat{g}}(1)$, the equivalence of (2) and (3) is immediate. Furthermore, the conformal invariance of ker $P_{k, g}$ as a space of conformal densities of weight $k-\frac{n}{2} \neq 0$ implies that $\operatorname{ker} P_{k, g}$ contains a nowhere vanishing function if and only if there is a metric $\hat{g}$ in the conformal class [g] such that $\operatorname{ker} P_{k, \hat{g}}(1)=0$. This proves the equivalence of (1) and (3) and completes the proof.

Remark 5.9. In the recent paper, Fardoun and Regbaoui [34] study the $Q$-curvature prescription problem on even-dimensional conformal classes for which the kernel of the critical GJMS operator is nontrivial kernel (i.e., it contains nonconstant functions). In particular, they give sufficient conditions for the convergence of the $Q$-curvature flow in terms of nodal domains of null-eigenfunctions of the critical GJMS operator.

\section{The Yamabe and Paneitz Operators on Heisenberg Manifolds}

In this section, we explicitly compute the eigenvalues and nodal sets of the Yamabe and Paneitz operators on Heisenberg manifolds.

\subsection{The setup}

Let $\mathbb{H}_{d}$ be the $(2 d+1)$-dimensional Heisenberg group, that is, the 2-nilpotent subgroup of $\mathrm{GL}_{d+2}(\mathbb{R})$ of unipotent matrices. Thus, any $A \in \mathbb{H}_{d}$ is of the form,

$$
A=\left(\begin{array}{ccc}
1 & x & t \\
0 & 1 & y^{T} \\
0 & 0 & 1
\end{array}\right), \quad x, y \in \mathbb{R}^{d}, \quad t \in \mathbb{R}
$$

We shall use coordinates $x=\left(x_{1}, \ldots x_{d}\right), y=\left(y_{1}, \ldots, y_{d}\right)$ and $t$ as above to represent an element of $\mathbb{H}_{d}$. 
Let $r=\left(r_{1}, \ldots, r_{d}\right) \in \mathbb{Z}^{d}$ be such that $r_{j} \mid r_{j+1}$ for $j=1, \ldots, d-1$ and consider the lattice subgroup

$$
\Gamma_{r}=\left\{\left(\begin{array}{ccc}
1 & x & t \\
0 & 1 & y^{T} \\
0 & 0 & 1
\end{array}\right) ; x \in \mathbb{Z}^{d}, y \in r_{1} \mathbb{Z} \times \cdots \times r_{d} \mathbb{Z}, t \in \mathbb{Z}\right\} .
$$

In addition, consider the quotient manifold,

$$
M:=\Gamma_{r} \backslash \mathbb{H}_{d}
$$

This is a compact manifold with fundamental group $\mathbb{H}_{d}$. Moreover, a fundamental domain for this quotient is

$$
D=[0,1)^{d} \times\left[0, r_{1}\right) \times \cdots \times\left[0, r_{d}\right) \times[0,1)
$$

A tangent frame of $\mathbb{H}_{d}$ is provided by the left-invariant vector fields:

$$
X_{j}=\frac{\partial}{\partial x_{j}}, \quad Y_{j}=\frac{\partial}{\partial y_{j}}+x_{j} \frac{\partial}{\partial t}, \quad T=\frac{\partial}{\partial t}
$$

where $j$ ranges over $1, \ldots, d$. The standard contact form on $\mathcal{H}_{d}$ is the left-invariant 1 -form given by

$$
\theta:=\mathrm{d} t-\sum_{1 \leq j \leq d} x_{j} \mathrm{~d} y_{j}
$$

Note that the 1 -forms $\mathrm{d} t$ and $\mathrm{d} x_{j}$ and $\mathrm{d} y_{j}$ are left-invariant too.

Let $s>0$. We endow $\mathbb{H}_{d}$ with the left-invariant metric

$$
g_{s}:=\sum_{1 \leq j \leq d} \mathrm{~d} x_{j} \otimes \mathrm{d} x_{j}+\sum_{1 \leq j \leq d} s^{-2} \mathrm{~d} y_{j} \otimes \mathrm{d} y_{j}+s^{2 d} \theta \otimes \theta
$$

This is the type of left-invariant Riemannian metrics considered in [42]. Note that the volume of $M$ with respect to $g_{s}$ is independent of $s$ and is equal to

$$
\left|\Gamma_{r}\right|:=r_{1} \cdots r_{d}
$$

Observe that $\left\{X_{j}, s Y_{j}, s^{-d} T\right\}$ is an orthonormal frame for this metric and $\operatorname{det} g_{s}=1$. Therefore, the Laplace operator $\Delta_{g_{s}}$ on $\mathbb{H}_{d}$ is given by

$$
\Delta_{g_{s}}=-\sum_{1 \leq j \leq d}\left(X_{j}^{2}+s^{2} Y_{j}^{2}\right)-s^{-2 d} T^{2} .
$$


In addition, it follows from the results of [59] that the Ricci tensor of $g_{s}$ (seen as a $(0,2)$-tensor) is given by

$$
\begin{aligned}
\operatorname{Ric}_{g_{s}} & =-\frac{1}{2} s^{2 d+2} \sum_{1 \leq j \leq d}\left(\mathrm{~d} x_{j} \otimes \mathrm{d} x_{j}+s^{-2} \mathrm{~d} y_{j} \otimes \mathrm{d} y_{j}\right)+\frac{d}{2} s^{4 d+2} \theta \otimes \theta \\
& =-\frac{1}{2} s^{2 d+2} g+\frac{(d+1)}{2} s^{4 d+2} \theta \otimes \theta
\end{aligned}
$$

We then get the following formula for the scalar curvature,

$$
R_{g_{s}}=-\frac{d}{2} s^{2 d+2}
$$

\subsection{Spectral resolution of the Yamabe operator}

As all the objects above are left-invariant, they descend to $M=\Gamma \backslash \mathbb{H}_{d}$. In particular, the Laplacian $\Delta_{g_{s}}$ on $M$ is given by (6.2), where the vector fields $X_{j}, Y_{j}$, and $T$ are meant as vector fields on $M$. Therefore, combining this with the above formula for the scalar curvature, we obtain the following proposition.

Proposition 6.1. The Yamabe operator of $\left(M, g_{s}\right)$ is given by

$$
P_{1, g_{s}}=-\sum_{1 \leq j \leq d}\left(X_{j}^{2}+s^{2} Y_{j}^{2}\right)-s^{-2 d} T^{2}-\frac{2 d-1}{16} s^{2 d+2} .
$$

The spectral resolution of $\Delta_{g_{s}}$ on $M$ is intimately related to the representation theory of $\mathbb{H}_{d}$. Indeed, the right-action of $\mathbb{H}_{d}$ on itself descends to a right-action on $M$, and hence the right-regular representation descends to the unitary representation

$$
\rho: \mathbb{H}_{d} \longrightarrow L^{2}(M)
$$

This representation can be decomposed into irreducible representations as follows.

Recall that the irreducible representations of $\mathbb{H}_{d}$ are of two types:

(i) The characters $\chi_{(\xi, \eta)}: \mathbb{H}_{d} \rightarrow \mathbb{C},(\xi, \eta) \in \mathbb{R}^{d} \times \mathbb{R}^{d}$, defined by

$$
\chi_{(\xi, \eta)}(x, y, t)=\mathrm{e}^{2 \mathrm{i} \pi(\xi \cdot x+\eta \cdot y)} .
$$


22 Y. Canzani et al.

(ii) The infinite-dimensional representations $\pi_{h}: \mathbb{H}_{d} \rightarrow \mathcal{L}\left(L^{2}\left(\mathbb{R}^{d}\right)\right), h \in \mathbb{R}^{*}$, given by

$$
\left[\pi_{h}(x, y, t) f\right](\xi):=\mathrm{e}^{2 \mathrm{i} \pi h(t+y \cdot \xi)} f(\xi+x) \quad \forall f \in L^{2}\left(\mathbb{R}^{d}\right)
$$

We observe that for the characters $\chi_{(\xi, \eta)}$, we have

$$
X_{j} \chi_{(\xi, \eta)}=2 \mathrm{i} \pi \xi_{j} \chi_{(\xi, \eta)}, \quad Y_{j} \chi_{(\xi, \eta)}\left(Y_{j}\right)=2 \mathrm{i} \pi \eta_{j} \chi_{(\xi, \eta)}, \quad T \chi_{(\xi, \eta)}=0
$$

For the representations $\pi_{h}$, we have

$$
\mathrm{d} \pi_{h}\left(X_{j}\right)=\frac{\partial}{\partial \xi_{j}}, \quad \mathrm{~d} \pi_{h}\left(Y_{j}\right)=2 \mathrm{i} \pi h \xi_{j}, \quad \mathrm{~d} \pi_{h}(T)=2 \mathrm{i} \pi h .
$$

For $n \in \mathbb{Z}$, define

$$
\mathcal{H}_{n}:=\left\{f \in L^{2}(M) ; f(x, y, t)=\mathrm{e}^{2 \mathrm{in} \pi t} g(x, y)\right\}
$$

In particular, $\mathcal{H}_{0}$ is the space of functions that do not depend on the $t$-variable.

Define

$$
\begin{aligned}
\Lambda & =\mathbb{Z}^{d} \times\left(\left(r_{1} \mathbb{Z}\right) \times \cdots \times\left(r_{d} \mathbb{Z}\right)\right), \\
\Lambda^{\prime} & =\left\{(\mu, v) \in \mathbb{R}^{d} \times \mathbb{R}^{d} ; \mu \cdot x+v \cdot y \in \mathbb{Z} \forall(x, y) \in \Lambda\right\} .
\end{aligned}
$$

Note that $\Lambda$ is the lattice of $\mathbb{R}^{2 d}$ given by the image of $\Gamma$ under the projection $(x, y, t) \rightarrow$ $(x, y)$. The set $\Lambda^{\prime}$ is its dual lattice.

Let $n \in \mathbb{Z}^{*}$. We set

$$
\begin{aligned}
\mathcal{A}_{n} & :=\left\{a=\left(a_{1}, \ldots, a_{d}\right) \in \mathbb{R}^{d} ; a_{j} \in\left\{0, \frac{1}{|n|}, \ldots, \frac{|n|-1}{|n|}\right\}\right\} \\
\mathcal{B} & :=\left\{b=\left(b_{1}, \ldots, b_{d}\right) ; b_{j} \in\left\{0, \frac{1}{r_{j}}, \ldots, \frac{r_{j}-1}{r_{j}}\right\}\right\}
\end{aligned}
$$

For $a \in \mathcal{A}_{n}$ and $b \in \mathcal{B}$, we define the operator $W_{n}^{a, b}: L^{2}\left(\mathbb{R}^{d}\right) \rightarrow L^{2}(M)$ by

$$
W_{n}^{a, b} f(x, y, t)=\mathrm{e}^{2 \mathrm{i} \pi n t} \sum_{k \in \mathbb{Z}_{d}} f(x+k+a+b) \mathrm{e}^{2 \mathrm{i} \pi n(k+a+b) \cdot y} .
$$


This is an isometry from $L^{2}\left(\mathbb{R}^{d}\right)$ into $\mathcal{H}_{n}$ (see [41]). We then let

$$
\mathcal{H}_{n}^{a, b}:=W_{n}^{a, b}\left(L^{2}\left(\mathbb{R}^{d}\right)\right)
$$

Proposition 6.2 (Brezin [18]). We have the following orthogonal decompositions:

$$
\begin{gathered}
L^{2}(M)=\bigoplus_{n \in \mathbb{Z}} \mathcal{H}_{n}, \\
\mathcal{H}_{0}=\bigoplus_{(\mu, \nu) \in \Lambda^{\prime}} \mathbb{C} \chi_{(\mu, \nu)}, \quad \mathcal{H}_{n}=\bigoplus_{\substack{a \in \mathcal{A}_{n} \\
b \in \mathcal{B}}} \mathcal{H}_{n}^{a, b}, \quad n \neq 0 .
\end{gathered}
$$

Moreover, each operator $W_{n}^{a, b}$ is an intertwining operator from $\pi_{n}$ to the regular representation $\rho$. In particular, the multiplicity of $\pi_{n}$ in $\rho$ is equal to $|n|^{d} \mid r_{1} \cdots r_{d}=$ $|n|^{d}\left|\Gamma_{r}\right|$.

Thanks to this result the spectral analysis of $\Delta_{g_{s}}$ on $L^{2}(M)$ reduces to the spectral analysis on each of the irreducible subspaces $\mathbb{C} \chi_{(\mu, \nu)}$ and $\mathcal{H}_{n}^{a, b}$.

Let $(\xi, \eta) \in \Lambda^{\prime}$. Then, from (6.2) and (6.7), we see that

$$
\Delta_{g_{s}} \chi_{(\xi, \eta)}=4 \pi^{2}\left(|\xi|^{2}+s^{2}|\eta|^{2}\right) \chi_{(\xi, \eta)} .
$$

That is, $\chi_{(\xi, \eta)}$ is an eigenfunction of $\Delta_{g_{s}}$ w.r.t. the eigenvalue $\lambda=4 \pi^{2}\left(|\xi|^{2}+s^{2}|\eta|^{2}\right)$.

Let $n \in \mathbb{Z}^{*}$. Using (6.2) and (6.8), we obtain

$$
\mathrm{d} \pi_{n}\left(\Delta_{g_{s}}\right)=\sum_{1 \leq j \leq d}\left(-\partial_{\xi_{j}}^{2}+4 n^{2} s^{2} \pi^{2} \xi_{j}^{2}\right)+4 n^{2} s^{-2 d} \pi^{2} .
$$

Under the change of variable $\eta_{j}=\sqrt{2 \pi|n| s} \xi_{j}$ this becomes

$$
2 \pi|n| s \sum_{1 \leq j \leq d}\left(-\partial_{\eta_{j}}^{2}+\eta_{j}^{2}\right)+4 n^{2} s^{-2 d_{\pi^{2}}} .
$$

Recall that on $\mathbb{R}$ that an orthogonal eigenbasis of $L^{2}(\mathbb{R})$ for the harmonic oscillator $-\frac{\mathrm{d}^{2}}{\mathrm{~d} v^{2}}+v^{2}$ is given by the Hermite functions, $h_{k}(v), k \in \mathbb{N}_{0}$, such that

$$
h_{k}(v):=(-1)^{k} \frac{\mathrm{d}^{k}}{\mathrm{~d} v^{k}} \mathrm{e}^{-\frac{1}{2} v^{2}}, \quad\left(-\frac{\mathrm{d}^{2}}{\mathrm{~d} v^{2}}+v^{2}\right) h_{k}(v)=(1+2 k) h_{k}(v)
$$

Note that $\mathrm{e}^{\frac{1}{2} v^{2}} h_{k}(v)$ is a polynomial of degree $k$. 
24 Y. Canzani et al.

From all this, we deduce that an orthogonal basis of eigenfunctions of $\mathrm{d} \pi_{n}\left(\Delta_{g_{s}}\right)$ is given by the functions,

$$
f_{\alpha}(\xi):=\prod_{1 \leq j \leq d} h_{\alpha_{j}}\left(\sqrt{2 \pi|n| s} \xi_{j}\right), \quad \alpha \in \mathbb{N}_{0}^{d}
$$

in such way that

$$
\mathrm{d} \pi_{n}\left(\Delta_{g_{s}}\right) f_{\alpha}=\left(2 \pi|n| s(d+2|\alpha|)+4 n^{2} s^{-2 d_{\pi^{2}}}\right) f_{\alpha} .
$$

Note that each eigenvalue $2 \pi|n| s(d+2|\alpha|)+4 n^{2} s^{-2 d} \pi^{2}$ occurs with multiplicity $\left(\begin{array}{c}|\alpha|+d-1 \\ d-1\end{array}\right)$.

As $W_{n}^{a, b}$ intertwines from $\pi_{n}$ to the regular representation $\rho$, we see that an orthogonal eigenbasis of $\mathcal{H}_{n}^{a, b}$ for $\Delta_{g_{s}}$ is given by the functions,

$$
\begin{aligned}
W_{n}^{a, b} f_{\alpha}(x, y, t) & =\mathrm{e}^{2 \mathrm{i} n \pi t} \sum_{k \in \mathbb{Z}^{d}} f_{\alpha}(x+k+a+b) \mathrm{e}^{2 \mathrm{i} n \pi(k+a+b) \cdot y} \\
& =\mathrm{e}^{2 \mathrm{i} n \pi t} \sum_{k \in \mathbb{Z}^{d}} \prod_{1 \leq j \leq d} h_{\alpha_{j}}\left(\sqrt{2 \pi|n| s}\left(x_{j}+k_{j}+a_{j}+b_{j}\right)\right) \mathrm{e}^{2 \mathrm{i} n \pi\left(k_{j}+a+b\right) y_{j}} \\
& =\mathrm{e}^{2 \mathrm{i} n \pi t} \prod_{1 \leq j \leq d}\left\{\sum_{k \in \mathbb{Z}} h_{\alpha_{j}}\left(\sqrt{2 \pi|n| s}\left(x_{j}+k_{j}+a_{j}+b_{j}\right)\right) \mathrm{e}^{2 \mathrm{i} n \pi(k+a+b) y_{j}}\right\} .
\end{aligned}
$$

Each $W_{n}^{a, b} f_{\alpha}$ is an eigenfunction for the eigenvalue $2 \pi|n| s(d+2|\alpha|)+4 n^{2} s^{-2 d_{\pi}}$. This eigenvalue has multiplicity $|n|^{d} r_{1} \cdots r_{d}\left(\begin{array}{c}|\alpha|+d-1 \\ d-1\end{array}\right)$ in $\mathcal{H}_{n}$.

As it turns out, for $\alpha=0$, the function $W_{n}^{a, b} f_{0}$ can be expressed in terms of Jacobi's theta function,

$$
\vartheta(z, \tau):=\sum_{k \in \mathbb{Z}} \mathrm{e}^{\mathrm{i} \pi k^{2} \tau} \mathrm{e}^{2 \mathrm{i} \pi k z}, \quad z, \tau \in \mathbb{C}, \quad \Im \tau>0
$$

If $\alpha=0$, then $h_{\alpha_{j}}(v)=h_{0}(v)=\mathrm{e}^{-\frac{1}{2} v^{2}}$ for $j=1, \ldots, d$. Moreover, for $u>0$ and $v, c \in \mathbb{R}$, we have

$$
\begin{aligned}
\sum_{k \in \mathbb{Z}} h_{0}(\sqrt{2 \pi|n| s}(u+k)) \mathrm{e}^{2 \mathrm{in} n \pi(k+c) v} & =\sum_{k \in \mathbb{Z}} \mathrm{e}^{-\pi|n| s(u+k)^{2}} \mathrm{e}^{2 \mathrm{i} n \pi(k+c) v} \\
& =\mathrm{e}^{2 \mathrm{i} n \pi c v} \sum_{k \in \mathbb{Z}} \mathrm{e}^{-\pi|n| s\left(u^{2}+2 k u+u^{2}\right)} \mathrm{e}^{2 \mathrm{i} n \pi k v}
\end{aligned}
$$




$$
\begin{aligned}
& =\mathrm{e}^{-\pi|n| s u^{2}} \mathrm{e}^{2 \mathrm{i} n \pi c v} \sum_{k \in \mathbb{Z}} \mathrm{e}^{-\pi|n| s u^{2}} \mathrm{e}^{2 \mathrm{i} n \pi k(v \pm i s u)} \\
& =\mathrm{e}^{-\pi|n| s u^{2}} \mathrm{e}^{2 \mathrm{i} n \pi c v} \vartheta(v \pm i s u, i|n| s)
\end{aligned}
$$

where \pm is the sign of $n$. Applying this equality to $u=x_{j}+a_{j}+b_{j}, v=y_{j}$, and $c=a_{j}+b_{j}$ and using (6.9) we obtain

$$
\begin{aligned}
& W_{n}^{a, b} f_{0}(x, y, t) \\
& =\mathrm{e}^{2 \mathrm{i} n \pi t} \prod_{1 \leq j \leq d}\left\{\mathrm{e}^{-\pi|n| s\left(x_{j}+a_{j}+b_{j}\right)^{2}} \mathrm{e}^{2 \mathrm{i} n \pi\left(a_{j}+b_{j}\right) v} \vartheta\left(y_{j} \pm i s\left(x_{j}+a_{j}+b_{j}\right), i|n| s\right)\right\} \\
& =\mathrm{e}^{2 \mathrm{in} n t t} \mathrm{e}^{-\pi|n| s|x+a+b|^{2}} \mathrm{e}^{2 \mathrm{i} n \pi(a+b) \cdot v} \prod_{1 \leq j \leq d} \vartheta\left(y_{j} \pm i s\left(x_{j}+a_{j}+b_{j}\right), i|n| s\right) .
\end{aligned}
$$

Combining the previous discussion with Proposition 6.1, we obtain the following proposition.

Proposition 6.3 (Compare [42, Theorem 3.3]). Assume that $M=\Gamma_{r} \backslash \mathbb{H}_{d}$ is equipped with the metric $g_{s}$ given by (6.1).

(1) An orthogonal eigenbasis of $L^{2}(M)$ for the Yamabe operator $P_{1, g_{s}}$ is given by the join

$$
\left\{\chi_{(\xi, \eta)} ;(\xi, \eta) \in \Lambda^{\prime}\right\} \cup\left\{W_{n}^{a, b} f_{\alpha} ;(n, a, b, \alpha) \in \mathbb{Z}^{*} \times \mathcal{A}_{n} \times \mathcal{B} \times \mathbb{N}_{0}^{d}\right\}
$$

The characters $\chi_{(\xi, \eta)}$ are given by (6.5). The functions $W_{n}^{a, b} f_{\alpha}$ are given by (6.9), which reduces to (6.10) when $\alpha=0$.

(2) Each character $\chi_{(\xi, \eta)}$ is an eigenfunction of $P_{1, g_{s}}$ with eigenvalue

$$
\lambda(\xi, \eta):=4 \pi^{2}\left(|\xi|^{2}+s^{2}|\eta|^{2}\right)-\frac{2 d-1}{16} s^{2 d+2} .
$$

(3) Each function $W_{n}^{a, b} f_{\alpha}$ is an eigenfunction of $P_{1, g_{s}}$ with eigenvalue

$$
\mu(n,|\alpha|):=2 \pi|n| s(d+2|\alpha|)+4 n^{2} s^{-2 d} \pi^{2}-\frac{2 d-1}{16} s^{2 d+2} .
$$

This eigenvalue has multiplicity $\left.|n|^{d}\left|\Gamma_{r}\right|^{(\alpha \mid+d-1} \begin{array}{c}(-1 \\ d-1\end{array}\right)$ in $\mathcal{H}_{n}$.

Remark 6.4 (See also [42, Theorem 3.3]). The above considerations also provides us with a spectral resolution of the Laplacian $\Delta_{g_{s}}$. More precisely, we see that 
(i) Each $\chi_{(\xi, \eta)}$ is an eigenfunction of $\Delta_{g_{s}}$ with eigenvalue

$$
\lambda_{0}(\xi, \eta):=4 \pi^{2}\left(|\xi|^{2}+s^{2}|\eta|^{2}\right)
$$

(ii) Each function $W_{n}^{a, b} f_{\alpha}$ is an eigenfunction of $P_{1, g_{s}}$ with eigenvalue

$$
\mu_{0}(n,|\alpha|):=2 \pi|n| s(d+2|\alpha|)+4 n^{2} s^{-2 d} \pi^{2} .
$$

\subsection{Nodal sets and negative eigenvalues of $P_{1, g_{s}}$}

We observe that an eigenfunction $W_{n}^{a, b} f_{\alpha}$ lies in the nullspace of $P_{1, g_{s}}$ if and only if

$$
2 \pi|n| s(d+2|\alpha|)+4 n^{2} s^{-2 d} \pi^{2}-\frac{2 d-1}{16} s^{2 d+2}=0 .
$$

If we multiply both sides of the equation by $-s^{2 d}$ and set $v=s^{2 d+1}$, then this equation becomes the quadratic equation $\frac{2 d-1}{16} v^{2}-2 \pi|n|(d+2|\alpha|) v-4 n^{2} \pi^{2}=0$, whose unique positive root is $v=\frac{8 \pi|n|}{2 d-1}\left(2(d+2|\alpha|)+\sqrt{4(d+2|\alpha|)^{2}+2 d-1}\right)$. Therefore, $W_{n}^{a, b} f_{\alpha}$ lies in the nullspace of $P_{1, g_{s}}$ if and only if

$$
s^{2 d+1}=\frac{8 \pi|n|}{2 d-1}\left(2(d+2|\alpha|)+\sqrt{4(d+2|\alpha|)^{2}+2 d-1}\right) .
$$

Let us give a lower bound for $v_{1}\left(g_{s}\right)$, that is, the number of negative eigenvalues of $P_{1, g_{s}}$. An eigenvalue $\mu(n,|\alpha|)=2 \pi|n| s(d+2|\alpha|)+4 n^{2} s^{-2 d_{\pi}}-\frac{2 d-1}{16} s^{2 d+2}$ is negative if and only if

$$
s^{2 d+1}>\frac{8 \pi|n|}{2 d-1}\left(2(d+2|\alpha|)+\sqrt{4(d+2|\alpha|)^{2}+2 d-1}\right) .
$$

In particular, for every integer $n$ such that $\frac{8 \pi|n|}{2 d-1}\left(2 d+\sqrt{4 d^{2}+2 d-1}\right)<s^{2 d+1}$, the eigenvalue $\mu(n, 0)$ is negative. Moreover, such an eigenvalue occurs with multiplicity $|n|^{d}\left|\Gamma_{r}\right|$. Therefore, we obtain the following proposition.

Proposition 6.5. There is a constant $c_{d}>0$ depending on $d$, but not on the sequence $r=\left(r_{1}, \ldots, r_{d}\right)$, such that

$$
v_{1}\left(g_{s}\right) \geq c_{d}\left|\Gamma_{r}\right| s^{2 d+2} \quad \forall s>0 .
$$

In particular, for every integer $m \in \mathbb{N}$, the Yamabe operator $P_{1, g_{s}}$ has at least $m$ negative eigenvalues as soon as $s$ is large enough. 
Suppose now that $s^{2 d+1}=\frac{8 \pi}{2 d-1}\left(2 d+\sqrt{4 d^{2}+2 d-1}\right)$, that is, (6.15) holds for $n= \pm 1$ and $\alpha=0$. Note that $\mathcal{A}_{ \pm 1}=\{0\}$, so $\operatorname{ker} P_{1, g_{s}} \cup \mathcal{H}_{0}^{\perp}$ is spanned by the functions $W_{ \pm 1}^{0, b} f_{0}$ given by (6.10) where $b$ ranges over $\mathcal{B}$. Furthermore, the transcendence of $\pi$ implies that if $s^{2 d+1}=\frac{8 \pi}{2 d-1}\left(2 d+\sqrt{4 d^{2}+2 d-1}\right)$, then no element $(\xi, \eta) \in \Lambda^{\prime}$ satisfies $\lambda(\xi, \eta)=$ $|\xi|^{2}+s^{2}|\eta|^{2}-\frac{2 d-1}{16} s^{2 d+2}=0$, that is, no character $\chi_{(\xi, \eta)}$ is contained in $\operatorname{ker} P_{1, g_{s}}$. Thus the functions $W_{ \pm 1}^{0, b} f_{0}, b \in \mathcal{B}$, form an orthogonal eigenbasis of $\operatorname{ker} P_{1, g_{s}}$.

Let us now look at the nodal sets of the eigenfunctions $W_{ \pm 1}^{0, b} f_{0}$. It follows from (6.10) that $W_{ \pm 1}^{0, b} f_{0}(x, y, t)=0$ if and only if

$$
\vartheta\left(y_{j} \pm \mathrm{i} s\left(x_{j}+b_{j}\right), \mathrm{is}\right)=0 \text { for some } j \in\{1, \ldots, d\} .
$$

Moreover, by Jacobi's triple product formula,

$$
\vartheta(z, 1 s)=\prod_{m=1}^{\infty}\left(1-\mathrm{e}^{-2 m \pi s}\right)\left(1+\mathrm{e}^{2 \mathrm{i} \pi z} \mathrm{e}^{-(2 m-1) \pi s}\right)\left(1+\mathrm{e}^{-2 \mathrm{i} \pi z} \mathrm{e}^{-(2 m-1) \pi s}\right) .
$$

Thus, for $z=v+\mathrm{i} s u, u, v \in \mathbb{R}$, we see that $\vartheta(z, 1 s)=0$ if and only if there is $m \in \mathbb{Z}$ such that $\mathrm{e}^{-(2 m-1) \pi s}=-\mathrm{e}^{2 \mathrm{i} \pi z}=\mathrm{e}^{-2 \pi s u+i \pi(2 v+1)}$, that is, $u$ and $v$ are contained in $\frac{1}{2}+\mathbb{Z}$. Applying this to $u=x_{j}+b_{j}$ and $v= \pm y_{j}$ with $\left(x_{j}, y_{j}\right) \in[0,1) \times\left[0, r_{j}\right)$ and $b_{j} \in\left\{0, r_{j}^{-1}, \ldots, 1-r_{l}^{-1}\right\}$ it not hard to deduce that

$$
\vartheta\left(y_{j} \pm \mathrm{i} s\left(x_{j}+b_{j}\right), \mathrm{i} s\right)=0 \Longleftrightarrow\left\{\begin{array}{l}
x_{j}= \pm\left(b_{j}-\frac{1}{2}\right)-\left[ \pm\left(b_{j}-\frac{1}{2}\right)\right], \\
y_{j} \in\left\{\frac{1}{2}, \frac{3}{2}, \ldots, r_{j}-\frac{1}{2}\right\},
\end{array}\right.
$$

where [.] is the floor function. (Here $x_{j}= \pm\left(b_{j}-\frac{1}{2}\right)-\left[ \pm\left(b_{j}-\frac{1}{2}\right)\right]$ is the only element of $[0,1)$ such that $\pm\left(x_{j}+b_{j}\right)$ is a half-integer.) Combining with (6.16) enables us to get the nodal set of $W_{ \pm 1}^{0, b} f_{0}$.

Summarizing the previous discussion, we have proved the following proposition.

Proposition 6.6. Let $s$ be the $(2 d+1)$ th root of $\frac{8 \pi}{2 d-1}\left(2 d+\sqrt{4 d^{2}+2 d-1}\right)$.

(1) The functions $W_{ \pm 1}^{0, b} f_{0}, b \in \mathcal{B}$, form an orthogonal basis of ker $P_{1, g_{s}}$.

(2) The nodal set of $W_{ \pm 1}^{0, b} f_{0}$ is given by the join

$$
\bigcup_{\substack{1 \leq j \leq d \\ 1 \leq l \leq r_{j}}}\left\{(x, y, t) \in M ; x_{j}= \pm\left(b_{j}-\frac{1}{2}\right)-\left[ \pm\left(b_{j}-\frac{1}{2}\right)\right], y_{j}=l-\frac{1}{2}\right\} .
$$


Remark 6.7. The nodal sets of the eigenfunctions $W_{ \pm 1}^{0, b} f_{0}$ are submanifolds of codimension 2 in $M$.

Null eigenvectors and negative eigenvalues can also occur from the characters $\chi_{(\xi, \eta)}$. To simplify the discussion we assume that $d=1$ and $r_{1}=1$.

The eigenfunction $\chi_{(\xi, \eta)}$ is in the kernel of $P_{1, g_{s}}$ if and only if $\lambda(\xi, \eta)=0$ which, according to Proposition 6.3, is equivalent to

$$
4 \pi^{2}\left(|\xi|^{2}+s^{2}|\eta|^{2}\right)=\frac{2 d-1}{16} s^{2 d+2}
$$

Although $\chi_{(\xi, \eta)}$ takes values in $\mathbb{S}^{1}$, we observe that $\chi_{(\xi, \eta)}$ is a null eigenvector if and only if so is $\chi_{(-\xi,-\eta)}=\overline{\chi(\xi, \eta)}$. Therefore, nodal sets to consider are $\mathcal{N}\left(\Re \chi_{\xi, \eta}\right)$ and $\mathcal{N}\left(\Im \chi_{\xi, \eta}\right)$. Note they are of the form $\mathcal{N}(x, y) \times[0,1]$ where the last factor corresponds to the $t$ coordinate.

As $r_{1}=1$, we see that $\xi$ and $\eta$ are in $\mathbb{Z}$ in (6.17). Accordingly, the values of $s$ for which a function $\chi_{(\xi, \eta)}$ lies in the kernel of $P_{1, g_{s}}$ are given by

$$
s^{2} \in\left\{32 \pi^{2}\left(\eta^{2}+\sqrt{\eta^{4}+\xi^{4} /\left(16 \pi^{2}\right)}\right): \xi, \eta \in \mathbb{Z}\right\} .
$$

Here, the dimension of the kernel is equal to the number of the pairs $(\xi, \eta)$ for which Equation (6.17) has solutions for a given $s \in \mathbb{R}$. An elementary calculation shows that $\left(\xi_{1}, \eta_{1}, s\right)$ and $\left(\xi_{2}, \eta_{2}, s\right)$ can both be solutions of (6.17) if and only if $\xi_{1}= \pm \xi_{2}, \eta_{1}= \pm \eta_{2}$ (otherwise $\pi$ would be a root of a nontrivial algebraic equation). It follows that for solutions of (6.17) and (6.18) the values of $\xi^{2}$ and $\eta^{2}$ are fixed.

Accordingly, the dimension of the kernel of $P_{1, g_{s}}$ is equal to either two (when $(\xi, \eta)=(0, \pm b)$ or $(\xi, \eta)=( \pm a, 0)$ ) or four (when $(\xi, \eta)=( \pm a, \pm b), a \neq 0, b \neq 0)$. It is now easy to describe some of the corresponding nodal sets.

In case $\xi=0, \eta= \pm a, s=4 a, a \in \mathbb{N}$, the eigenfunction is of the form $\sin 2 \pi$ $(a y+\theta)$. Accordingly, up to translation in $y$, the nodal set is

$$
[0,1] \times\{k /(2 a): 0 \leq k \leq 2 a\} \times[0,1] .
$$

In case $\eta=0, \xi= \pm a^{2}, s=2 a, a \in \mathbb{N}$, the eigenfunction is of the form $\sin 2 \pi\left(a^{2} x+\right.$ $\theta)$. Accordingly, up to translation in $x$, the nodal set is

$$
\left\{k /\left(2 a^{2}\right): 0 \leq k \leq 2 a^{2}\right\} \times[0,1] \times[0,1] .
$$


Finally, in case $\xi= \pm a, \eta= \pm b, s^{2}=8\left(\eta^{2}+\sqrt{\eta^{4}+\xi^{4} / 4}\right) ; a \neq 0, b \neq 0$, there exist "product" eigenfunctions of the form $\sin 2 \pi\left(a x+\theta_{1}\right) \cdot \sin 2 \pi\left(b y+\theta_{2}\right)$ with nodal sets is a union of sets of the form (6.20) and (6.19). In addition, there exist eigenfunctions of the form $\sin 2 \pi(a x+b y+\theta)$, whose nodal sets (up to translation) have the form

$$
\left\{(x, y) \in[0,1]^{2}: 2(a x+b y) \in \mathbb{Z}\right\} \times[0,1] .
$$

\subsection{The Paneitz operator}

Let us now look at the Paneitz operator (2.3). We have

$$
P_{2, g_{s}}:=\Delta_{g_{s}}^{2}+\delta V d+\frac{2 d-3}{2}\left\{\frac{1}{4 d} \Delta_{g_{s}} R_{g_{s}}+\frac{2 d+1}{8(2 d)^{2}} R_{g}^{2}-2|S|^{2}\right\}
$$

where $S=\frac{1}{2 d-1}\left(\operatorname{Ric}_{g_{s}}-\frac{R_{g_{s}}}{2(2 d)} g_{s}\right)$ is the Schouten-Weyl tensor and $V$ is the tensor $(V=$ $\left.\frac{2 d-1}{2(2 d)} R_{g_{s}} g_{s}-4 S\right)$ acting on 1 -forms.

Using (6.3-6.4), we see that the Schouten-Weyl tensor is given by

$$
\begin{aligned}
S & =-\frac{3}{8(2 d-1)} s^{2 d+2} g_{s}+\frac{d+1}{2(2 d-1)} s^{4 d+2} \theta \otimes \theta \\
& =-\frac{3}{8(2 d-1)} s^{2 d+2} \sum_{1 \leq j \leq d}\left(\mathrm{~d} x_{j} \otimes \mathrm{d} x_{j}+s^{-2} \mathrm{~d} y_{j} \otimes \mathrm{d} y_{j}\right)+\frac{4 d+1}{8(2 d-1)} s^{2 d+2} \cdot s^{2 d} \theta \otimes \theta .
\end{aligned}
$$

Observing that $\left\{\mathrm{d} x_{j} \otimes \mathrm{d} x_{j}, s^{-2} \mathrm{~d} y_{j} \otimes \mathrm{d} y_{j}, s^{2 d} \theta \otimes \theta\right\}$ is an orthonormal family of $(0,2)$ tensors, we find that

$$
|S|^{2}=\frac{16 d^{2}+18 d+1}{64(2 d-1)^{2}} s^{4 d+4} .
$$

We then deduce that the constant coefficient of $P_{2}$ is equal to

$$
(2 d-3) \frac{(2 d+1)(2 d-1)^{2}-4\left(16 d^{2}+18 d+1\right)}{256(2 d-1)^{2}} s^{4 d+4} .
$$

The tensor $V$ is given by

$$
V=\frac{12-(2 d-1)^{2}}{8(2 d-1)} s^{2 d+2} g_{s}-\frac{2(d+1)}{2 d-1} s^{4 d+2} \theta \otimes \theta
$$


We need to look at $V$ as acting on 1-forms. The action of $g_{s}$ on 1-form is just the identity. The action of $s^{2 d} \theta \otimes \theta$ is the orthogonal projection onto the span of $\theta$. Thus,

$$
\delta V d=\frac{12-(2 d-1)^{2}}{8(2 d-1)} s^{2 d+2} \Delta_{g_{s}}+2 \frac{d+1}{2 d-1} s^{2} T^{2}
$$

Combining all this together, we obtain the following proposition.

Proposition 6.8. The Paneitz operator on $M$ for the metric $g_{s}$ is given by

$$
P_{2, g_{s}}=\Delta_{g_{s}}^{2}-c_{1}(d) s^{2 d+2} \Delta_{g_{s}}+2 \frac{d+1}{2 d-1} s^{2} T^{2}+c_{0}(d) s^{4 d+4}
$$

where we have set

$$
c_{0}(d):=(2 d-3) \frac{(2 d+1)(2 d-1)^{2}-4\left(16 d^{2}+18 d+1\right)}{256(2 d-1)^{2}} \quad \text { and } \quad c_{1}(d):=\frac{(2 d-1)^{2}-12}{8(2 d-1)}
$$

Observe that $T \chi_{(\xi, \eta)}=0$ and $T^{2} W_{n}^{a, b} f_{\alpha}=-2 n^{2} \pi^{2}$. Therefore, we can use the spectral resolution of Laplacian $\Delta_{g_{s}}$ given by Remark 6.4 and to get a spectral resolution of $P_{2, g_{s}}$.

Proposition 6.9. Assume $M=\Gamma_{r} \backslash \mathbb{H}_{d}$ is equipped with the metric $g_{s}$ given by (6.1).

(1) The family (6.11) for an orthogonal eigenbasis of $L^{2}(M)$ for $P_{2, g_{s}}$.

(2) Each character $\chi_{(\xi, \eta)}$ is an eigenfunction of $P_{2, g_{s}}$ with eigenvalue

$$
\lambda_{0}(\xi, \eta)^{2}-c_{1}(d) s^{2 d+2} \lambda_{0}(\xi, \eta)+c_{0}(d) s^{4 d+4}
$$

where $\lambda_{0}(\xi, \eta)$ is given by (6.12).

(3) Each function $W_{n}^{a, b} f_{\alpha}$ is an eigenfunction of $P_{2, g_{s}}$ with eigenvalue

$$
\mu_{0}(n,|\alpha|)^{2}-c_{1}(d) s^{2 d+2} \mu_{0}(n,|\alpha|)+c_{0}(d) s^{4 d+4}-4 \frac{d+1}{2 d-1} n^{2} \pi^{2} s^{2}
$$

where $\mu_{0}(\xi, \eta)$ is given by (6.12). This eigenvalue has multiplicity $|n|^{d}\left|\Gamma_{r}\right|\left(\begin{array}{c}|\alpha|+d-1 \\ d-1\end{array}\right)$ in $\mathcal{H}_{n}$.

Set $F_{n}(\mu ; s):=\mu^{2}-c_{1}(d) s^{2 d+2} \mu+c_{0}(d) s^{4 d+4}-4 \frac{d+1}{2 d-1} n^{2} \pi^{2} s^{2}$. Then Proposition 6.9 states that $W_{n}^{a, b} f_{\alpha}$ is an eigenfunction of $P_{2, g_{s}}$ with eigenvalue $F_{n}\left(\mu_{0}(n,|\alpha|) ; s\right)$. Moreover, 
$F_{n}(\mu ; s)$ is a quadratic polynomial in $\mu$ with discriminant

$$
\delta_{n}(d, s):=\delta_{0}(d) s^{4 d+4}+16 \frac{d+1}{2 d-1} n^{2} \pi^{2} s^{2}, \quad \delta_{0}(d)=c_{1}(d)^{2}-4 c_{0}(d)
$$

A computation shows that $\delta_{0}(d)=\frac{1}{4} \frac{4 d^{2}-7}{2 d-1}$, which is positive for $d \geq 2$.

Assume $d \geq 2$. Then $\delta_{n}(d, s) \geq \delta_{0}(d) s^{4 d+4}>0$, and so $F_{n}\left(\mu_{0}(n,|\alpha|) ; s\right)$ is a negative eigenvalue of $P_{2, g_{s}}$ if and only if

$$
\mu_{0}(n,|\alpha|)<\frac{1}{2}\left(c_{1}(d) s^{2 d+2}+\sqrt{\delta_{n}(d, s)}\right)
$$

As $\delta_{n}(d, s) \geq \delta_{0}(d) s^{4 d+4}$, we see that $\mu_{0}(n,|\alpha|)$ satisfies the above condition if $\mu_{0}(n,|\alpha|)<$ $\frac{1}{2}\left(c_{1}(d)+\sqrt{\delta_{0}(d) s^{4 d+4}}\right)$. That is,

$$
2 \pi|n| s(d+2|\alpha|)+4 n^{2} s^{-2 d_{\pi^{2}}}<\frac{1}{2}\left(c_{1}(d)+\sqrt{\delta_{0}(d)}\right) s^{2 d+2} .
$$

This is the same type of condition than that incurring from (6.14) for $W_{n} f_{\alpha}^{a, b}$ to produce a negative eigenvalue of the Yamabe operator $P_{1, g_{s}}$. Therefore, by using the same kind of arguments as that used to derive of Proposition 6.5, we obtain the following proposition.

Proposition 6.10. Assume $d \geq 2$. Then there is a constant $c_{d}>0$ depending on $d$, but not on the sequence $r=\left(r_{1}, \ldots, r_{d}\right)$, such that

$$
v_{2}\left(g_{s}\right) \geq c_{d}\left|\Gamma_{r}\right| s^{2 d+2} \quad \forall s>0 .
$$

In particular, for every integer $m \in \mathbb{N}$, the Paneitz operator $P_{2, g_{s}}$ has at least $m$ negative eigenvalues as soon as $s$ is large enough.

\section{Open Problems}

\subsection{Discriminant hypersurfaces in the space of conformal structures}

Let $M$ be a compact orientable Riemannian manifold. Denote by $\mathcal{M}$, the space of all Riemannian metrics on $M$. Then one can consider the action on $\mathcal{M}$ of the group $\mathcal{P}$ of (pointwise) conformal transformations (multiplication by positive functions), as well as of the group $\mathcal{D}$ of diffeomorphisms; we shall denote by $\mathcal{D}_{0}$, the subgroup of $\mathcal{D}$ of diffeomorphisms isotopic to identity. 
The objects considered in the paper are invariant under the action of $\mathcal{P}$, and equivariant with respect to the action of $\mathcal{D}$. Accordingly, it seems natural to consider our invariants as functions on the Teichmüller space of conformal structures

$$
\mathcal{T}_{M}=\frac{\mathcal{M} / \mathcal{P}}{\mathcal{D}_{0}}
$$

or on Riemannian moduli space of conformal structures

$$
\mathcal{R}_{M}=\frac{\mathcal{M} / \mathcal{P}}{\mathcal{D}}
$$

in the terminology of Fischer and Monkrief, [39, 40]. If $M$ is an orientable twodimensional manifold, then $\mathcal{T}_{M}$ (respectively, $\mathcal{R}_{M}$ ) is the usual Teichmüller (respectively, moduli) spaces. In [40], the space $\mathcal{T}_{M}$ for Haken 3-manifolds $M$ of degree 0 is proposed as a configuration space for a Hamiltonian reduction of Einstein's vacuum field equations.

\subsection{Dimension of the nullspace of a noncritical GJMS operator}

The Dirac operator is another important conformally invariant operator. Results of Maier [67] in dimension 3 and Amman et al. [3] in higher dimension show that, on a compact Riemannian spin manifold, and for a generic metric, the dimension of the nullspace of the Dirac operator is equal to the lower bound provided by the Atiyah-Singer index theorem. In particular, the nullspace of the Dirac operator is generically trivial when $n \in\{3,4,5,6,7\} \bmod 8$.

Let $k \in \mathbb{N}$ and further assume $k<\frac{n}{2}$ when $n$ is even. For the GJMS operator $P_{k}$ we make the following conjecture.

Conjecture A. For a generic conformal class in $\mathcal{T}_{M}$ the nullspace of $P_{k}$ is trivial.

This conjecture will be addressed in the sequel [21]. For a general (possibly nongeneric) conformal class, we mention the following conjecture due to Colin Guillarmou [53].

Conjecture B (Guillarmou). Assume $n$ odd. Then, for any conformal class in $\mathcal{T}_{M}$, there exists $C>0$ such that

$$
\operatorname{dim} \operatorname{ker} P_{k} \leq C k^{n} \quad \forall k \in \mathbb{N} \text {. }
$$




\subsection{Hypersurfaces in $\mathcal{T}_{M}, \mathcal{R}_{M}$, and rigidity of the nodal set}

Bearing in mind Conjecture A, we consider the discriminant hypersurface $\mathcal{H}_{k}$ (in $\mathcal{T}$ or $\mathcal{R})$ consisting of conformal classes with nontrivial nullspace ker $P_{k} \neq 0$.

Conjecture C. For a generic conformal class in $\mathcal{H}_{k}$, the nullspace of $P_{k}$ has dimension 1.

Note that when dimker $P_{k}=1$ the nodal set and the nodal domains are welldefined. We also observe that $\mathcal{H}_{k}$ contains all conformal classes of Ricci-flat metrics, since for a Ricci-flat metric $g$ Equation (2.5) shows that $P_{k, g}=\Delta_{g^{\prime}}^{k}$ and hence $\operatorname{ker} P_{k, g}$ is equal to the space of constant functions.

The following inverse (rigidity) problem seems natural:

Problem D. Let $g$ be a metric such that $\operatorname{dim} \operatorname{ker} P_{k, g}=1$. Does the nodal set $\mathcal{N}(\phi)$, $\phi \in \operatorname{ker} P_{k, g}$, determine the corresponding conformal class $[g] \in \mathcal{H}_{k}$ uniquely (up to diffeomorphisms)? In other words, do our invariants separate points in $\mathcal{H}_{k}$ ?

The following weaker (deformation rigidity) version of the previous problem also seems interesting:

Problem E. Let $g$ be a metric such that $\operatorname{dim} \operatorname{ker} P_{k, g}=1$. Does the nodal set $\mathcal{N}(\phi), \phi \in$ $\operatorname{ker} P_{k, g}$, determine locally the conformal class [g]? In other words, can we deform a conformal class without changing $\mathcal{N}(\phi)$ ?

We remark that it seems quite natural to consider Problems D and E on the spaces $\mathcal{T}_{M}$ and $\mathcal{R}_{M}$, since the action of $\mathcal{P}$ preserves the nodal sets, and their definition is equivariant with respect to the action of $\mathcal{D}$. The first natural step in this direction seems to be the following one.

Problem F. Let $g$ be a metric such that $\operatorname{dim} \operatorname{ker} P_{k, g} \geq 1$. Determine the tangent space $T_{g} \mathcal{H} \subset T_{g} \mathcal{M}$.

\subsection{Dimension of the nullspace of the critical GJMS operator}

Assume $n$ even. For the critical GJMS operator $P_{\frac{n}{2}}$ on a $n$-dimensional manifold with $n$ even, the constant function will always be in $\operatorname{ker} P_{\frac{n}{2}}$. 
Conjecture G. For a generic conformal class in $\mathcal{T}_{M}$, the nullspace of ker $P_{\frac{n}{2}}$ consists of constant functions.

This conjecture is true if $M$ admits an Einstein metric of positive scalar curvature.

We define the discriminant hypersurface $\mathcal{H}_{\frac{n}{2}}$ as the set of conformal classes for which the dimension of the nullspace of $P_{\frac{n}{2}}$ is at least 2 .

Conjecture H. For a generic conformal class in $\mathcal{H}_{\frac{n}{2}}$, the nullspace of $P_{\frac{n}{2}}$ has dimension 2.

It seems interesting to study the geometry and topology of the various $\mathcal{H}_{k}$ and their complements in the space of all conformal classes of Riemannian metrics on $M$.

\subsection{Negative eigenvalues and topology of spaces of metrics}

Recall that it was shown in Proposition 4.6 that on any compact manifold of dimension $n \geq 3$, for any $m>0$ there exist metrics $g$ for which the Yamabe operator $P_{1, g}$ has at least $m$ negative eigenvalues. We have also constructed examples of Riemannian manifolds for which there are analogous results for some higher order GJMS operators (cf. Theorems 4.8 and 6.10).

Problem I. Let $k>1$, and let $M$ be a compact manifold of dimension $n \geq 3$. Can we find for every $m \in \mathbb{N}$ a metric $g_{m}$ on $M$ such that $P_{k, g_{m}}$ has at least $m$ negative eigenvalues?

We remark that if the number of negative eigenvalues of $P_{k, g}, k>1$, is uniformly from bounded above for every metric $g$ on $M$, then the smallest such bound would be a topological invariant of $M$.

On Yamabe-negative manifolds (which do not admit metrics of nonnegative scalar curvature), we know that in every conformal class there exists at least one negative eigenvalue of $P_{1, g}$. For such manifolds, the following question formulated in [5] seems natural:

Problem J. Let $M$ be a Yamabe-negative compact manifold of dimension $n \geq 3$. Does there exist an integer $m_{0} \geq 2$ such that in every conformal class on $M$, the Yamabe operator $P_{1, g}$ has at least $m_{0}$ negative eigenvalues? 
It is known from the work of Gromov and Lawson $[52,77]$ that, on many manifolds of dimension $n \geq 5$ (and on some manifolds of dimension 4), the space of Yamabepositive metrics (with positive scalar curvature, or equivalently without negative eigenvalues of $P_{1, g}$ ) can have infinitely many connected components.

On the other hand, Lohkamp ([65]; see also [61]) showed that the space of metrics with negative scalar curvature is connected and has trivial homotopy groups. Therefore, the following seems natural:

Problem K. Let $M$ be a compact manifold of dimension $n \geq 3$. Given integers $k$ and $m$, describe the topology of the space of all metrics $g$ for which the GJMS operator $P_{k, g}$ has at most $m$ negative eigenvalues (i.e., $\left.\lambda_{m+1}\left(P_{k, g}\right) \geq 0\right)$. In particular, is that space connected?

\section{Acknowledgements}

This paper has its origin from discussions between D.J. and R.G. during the 2010 Banff workshop on geometric scattering theory. They wish to thank BIRS and the workshop organizers for their hospitality. R.P. wishes to thank McGill University for its hospitality during his various visits to Montreal. In addition, the authors wish to thank Alice Chang, Penfei Guan, Colin Guillarmou, Niky Kamram, Rafe Mazzeo, Peter Sarnak, Richard Schoen, and Paul Yang for useful discussions related to he subject matter of this paper.

\section{Funding}

This work was supported by Schulich Fellowship of McGill University (Canada) (to Y.C.), Marsden Grant 10-UOA-113 (New Zealand) (to R.G.), NSERC and FORNT grants and Dawson Fellowship of McGill University (Canada) (to D.J.) and by JSPS Grant-in-Aid (Japan) and Research Resettlement Fund of Seoul National University (Korea) (to R.P.).

\section{Appendix by A. Rod Gover and Andrea Malchiodi}

Noncritical o curvature prescription: Forbidden functions arising from nontrivial nullspace

\section{A.1 Background}

Some literature and background concerning curvature prescription was mentioned in Section 5. Concerning the problem of prescribing $Q=Q_{\frac{n}{2}, g}$ on even manifolds: in 
$[45,68]$, it was shown that if the manifold and conformal structure is such that the related critical GJMS operator $P_{\frac{n}{2}}$ has nontrivial kernel (i.e., it contains nonconstant functions), then global considerations show that large classes of functions cannot arise as the $Q$-curvature for some metric in the given conformal class. Due to the curious properties of Branson's $Q$-curvature it turns out that the arguments required in [45] are mainly of a linear or quadratic nature and benefit from a conformal invariant identified in $[13,14]$.

The conformal prescription problem for the other ("noncritical") $Q$-curvatures is rather different (having polynomial instead of exponential nonlinearities). Nevertheless, we work with the cases $k \neq \frac{n}{2}$ here (so we exclusively consider the noncritical $Q$ curvatures) and show that there are again global obstructions to prescription of certain functions, arising from the presence of nontrivial GJMS kernel. In the following, $k$ is an integer from the usual range for the GJMS operators except that we shall suppose henceforth that $2 k \neq n$ (so $k$ is a positive integer with $2 k \notin\{n, n+2, \ldots\}$ ).

Recall the expression (2.7) defining the $Q$-curvatures and that in the case $k=1$ we have $Q_{1}=R_{g} / 2(n-1)$, where $R_{g}$ is the usual scalar curvature. In general, the quantity $Q_{k}$ in (2.7) is called the order $2 k$ (noncritical) $Q$-curvature; for simplicity we shall refer to this as simply a $Q$-curvature. As in the body of the article, for $Q_{k}$, we may write $Q_{k, g}$ to emphasize the dependence on the metric $g$; we similarly treat related quantities.

\section{A.2 The problem}

The $Q$-prescription problem is described in (5.1). The partial differential equation governing this follows from the conformal transformation of the $P_{k}$ operator, as discussed in Section 2. We summarize the facts from there in a form convenient for our current purposes.

For the conformal transformation of $P_{k, g}$, we have

$$
P_{k, \hat{g}} \mathrm{e}^{\frac{2 k-n}{2} \omega} u=\mathrm{e}^{-\frac{2 k+n}{2} \omega} P_{k, g} u
$$

where $\hat{g}=\mathrm{e}^{2 \omega} g, \omega, u \in C^{\infty}(M, \mathbb{R})$. So if we take, in particular, $u$ to be the positive function $u=\mathrm{e}^{\frac{n-2 k}{2} \omega}$, then $\mathrm{e}^{\frac{2 k-n}{2} \omega} u=1$, and so we conclude

$$
P_{k, \hat{g}} 1=u^{\frac{n+2 k}{2 k-n}} P_{k, g} u
$$


Putting (2.7) and (A.2) together we obtain the nonlinear equation governing (5.1):

$$
\left(\delta S_{k, g} d+\frac{n-2 k}{2} Q_{k, g}\right) u=\frac{n-2 k}{2} Q_{k, \hat{g}} u^{\frac{n+2 k}{n-2 k}},
$$

where $u$ is an arbitrary positive function. This generalizes the well-known scalar curvature prescription equation which is the $k=1$ special case.

\section{A.3 Forbidden functions}

Denote by $\mathcal{C}$ a conformal class of metrics on $M$. We are interested in what functions we can, or cannot, land on with $Q_{k, \hat{g}}$, where $\hat{g} \in \mathcal{C}$. Let us fix $k$ and drop it from the notation. So henceforth $P=P_{k}$ and $Q=Q_{k}$ for some fixed $k$ with $2 k \in 2 \mathbb{Z} \backslash\{n, n+2, n+4, \ldots\}$.

A first obstruction one can obtain rather easily, as in [62], is that if for some $g \in \mathcal{C}$ $Q_{g}$ has a given sign, then it is not possible to prescribe a function with the opposite sign: this follows immediately by integrating (A.3). We notice first that for the case $k=1$ the sign of the Yamabe invariant

$$
\inf _{\hat{g} \in \mathcal{C}} \frac{\int R_{\hat{g}} \mathrm{~d} v_{\hat{g}}}{\left(\int \mathrm{d} v_{\hat{g}}\right)^{\frac{n-2}{n}}}
$$

coincides with the sign of the first eigenvalue of the conformal Laplacian and determines uniquely the possible sign of the scalar curvature for the metrics in $\mathcal{C}$. This is not the case in general for larger $k$, due to a lack of maximum principle.

We have next the following observation, more peculiar to the presence of a kernel. Here and subsequently, we write ker $P_{g}$ for the kernel (or nullspace) of $P_{g}$.

Proposition A.1. Consider a closed manifold $M$ equipped with a conformal structure $\mathcal{C}$, and let $g \in \mathcal{C}$. If $0 \neq u \in \operatorname{ker} P_{g}$, then $u$ is not in the range of $Q$. That is, $u \neq Q_{\hat{g}}$ for all $\hat{g} \in \mathcal{C}$.

Proof. Suppose, with a view to contradiction, that $\hat{g} \in \mathcal{C}$ and $Q_{\hat{g}}=u$. Since $g, \hat{g} \in \mathcal{C}$ we have $\hat{g}=\mathrm{e}^{2 \omega} g$ for some $\omega \in C^{\infty}(M, \mathbb{R})$.

Now from (A.1), if $u \in \operatorname{ker} P_{g}$, then $\mathrm{e}^{f} u \in \operatorname{ker} P_{\hat{g}}$, where $f=\frac{2 k-n}{2} \omega$. So, using that $P_{\hat{g}}$ is formally self-adjoint, it follows that for any function $v$

$$
\int u \mathrm{e}^{f} P_{\hat{g}} v \mathrm{~d} v_{\hat{g}}=0
$$


Thus, taking $v=1$, this shows

$$
0=\frac{2}{n-2 k} \int u \mathrm{e}^{f} P_{\hat{g}} 1 \mathrm{~d} v_{\hat{g}}=\int u \mathrm{e}^{f} Q_{\hat{g}} \mathrm{~d} v_{\hat{g}}=\int u \mathrm{e}^{f} u \mathrm{~d} v_{\hat{g}}=\int u^{2} \mathrm{e}^{f} \mathrm{~d} v_{\hat{g}}
$$

This is a contradiction since $u^{2} \mathrm{e}^{f}$ is a nonzero nonnegative function.

More generally essentially the same argument shows that we cannot have $Q_{\hat{g}}$ equal $s_{u}$, where the latter is any function that has the same or opposite strict sign as $u$ : suppose with a view to contradiction $Q_{\hat{g}}=s_{u}$. Then

$$
0=\frac{2}{n-2 k} \int u \mathrm{e}^{f} P_{\hat{g}} 1 \mathrm{~d} v_{\hat{g}}=\int u \mathrm{e}^{f} s_{u} \mathrm{~d} v_{\hat{g}}=\int \mathrm{e}^{f} u s_{u} \mathrm{~d} v_{\hat{g}}
$$

which is impossible. Thus, we have the following result.

Theorem A.2. Consider a closed manifold $M$ equipped with a conformal structure $\mathcal{C}$, and let $g \in \mathcal{C}$. Suppose there exists $u \in \operatorname{ker} P_{g} \backslash\{0\}$. Then for any function $s_{u}$ on $M$ with the same or opposite strict sign as $u, s_{u}$ is not in the range of $Q$. That is, $s_{u} \neq Q_{\hat{g}}$, for all $\hat{g} \in \mathcal{C}$.

Observe that if there exists $u$, as in the Theorem, then there is a huge class of functions satisfying the conditions on $s_{u}$ : for example, $\mathrm{e}^{f} u^{p}$ where $p$ is an odd positive integer and $f \in C^{\infty}(M, \mathbb{R})$. We record this for emphasis.

Corollary A.3. If $P_{g}$ has nontrivial kernel, then there is an infinite-dimensional space of functions disjoint from $\mathcal{R}(Q)$.

\section{A.4 Constraints on $\mathcal{R}(Q)$}

To prove Theorem A.2, we used that given $u \in \operatorname{ker} P_{g}$ then, for any $\hat{g} \in \mathcal{C}$, we have

$$
\int u \mathrm{e}^{f} Q_{\hat{g}} \mathrm{~d} v_{g}=0
$$

for some real function $f$, which depends on $\hat{g}$. In fact, if $\hat{g}=\mathrm{e}^{2 \omega} g$, then $f=\frac{n+2 k}{2} \omega$, but the details are not important. The key point here is that $\mathrm{e}^{f}$ is a strictly positive function, thus for $u$ nonzero the display captures some nontrivial constraint on $\mathcal{R}(Q)$ as demonstrated in Theorem A.2 and its Corollary. 
Given elements $u \in \operatorname{ker} P_{g}$ and $g \in \mathcal{C}$, consider the linear form $I_{u}^{g}: C^{\infty}(M, \mathbb{R}) \rightarrow \mathbb{R}$ defined by

$$
I_{u}^{g}(v)=\int u v \mathrm{~d} v_{g} \quad \forall v \in C^{\infty}(M, \mathbb{R}) .
$$

Now let us fix some $g \in \mathcal{C}$, and for the moment also fix some $u \in \operatorname{ker} P_{g}$. From (A.4), and the conformal transformation of the standard metric measure, we have that if $v=Q_{\hat{g}}$ for some $\hat{g} \in \mathcal{C}$, then there exists $g^{\prime} \in \mathcal{C}$ such that

$$
\int u v \mathrm{~d} v_{g^{\prime}}=0
$$

Thus

$$
\mathcal{R}(Q) \subseteq \bigcup_{g^{\prime} \in \mathcal{C}} \mathcal{Z}\left(I_{u}^{g^{\prime}}\right)
$$

where $\mathcal{Z}\left(I_{u}^{g^{\prime}}\right)$ denotes the kernel of the map $I_{u}^{g^{\prime}}$. This holds for all elements of ker $P_{g}$, thus we have

$$
\mathcal{R}(Q) \subseteq \bigcap_{u^{\prime} \in \operatorname{ker} P_{g}}\left[\bigcup_{g^{\prime} \in \mathcal{C}} \mathcal{Z}\left(I_{u^{\prime}}^{g^{\prime}}\right)\right] .
$$

By definition $\mathcal{R}(Q)$ depends only on the conformal structure. On the other hand we had fixed $g \in \mathcal{C}$ to describe the right-hand side here. A different choice would result in each of the elements $u^{\prime} \in \operatorname{ker} P_{g}$ being replaced by a positive function multiple $\mathrm{e}^{f} u^{\prime}$, with the same function $\mathrm{e}^{f}$ for all elements of ker $P_{g}$. Examining (A.5), we see that this factor $\mathrm{e}^{f}$ may be absorbed by moving to a conformally related measure. Since we average over all such in the right-hand side of (A.6) it is clear that in fact this function space is independent of $g$, and depends only on $\mathcal{C}$.

Since ker $P_{g}$ is finite-dimensional and $I_{u^{\prime}}^{g}$ is linear in its dependence on $u^{\prime} \in \operatorname{ker} P_{g^{\prime}}$, we obtain the following refinement of the above.

Theorem A.4. On a closed conformal manifold $(M, \mathcal{C})$ let $g \in \mathcal{C}$. Then

$$
\mathcal{R}(Q) \subseteq \mathcal{I}=\bigcap_{i=1}^{\ell}\left[\bigcup_{g^{\prime} \in C} \mathcal{Z}\left(I_{u_{i}}^{g^{\prime}}\right)\right],
$$

where $\ell=\operatorname{dim} \operatorname{ker} P_{g}$, and $u_{1}, \ldots, u_{\ell}$ is a basis for ker $P_{g}$. Furthermore, the function space $\mathcal{I}$ is independent of the choice of $g \in \mathcal{C}$ and the choice of basis $\left\{u_{1}, \ldots, u_{\ell}\right\}$. 
An important special case is prescribing constant $Q$-curvature. This is related to the Yamabe problem which seeks to find within a conformal class a metric with constant scalar curvature. Note that if there is a metric $g \in \mathcal{C}$ such that $Q_{g}=$ constant $\neq 0$, then it is clear from (A.5) that any nonzero element of ker $P_{g}$ must change sign on $M$. But this sign change property is independent of $g \in \mathcal{C}$. Thus, by contrapositive, we have the following.

Theorem A.5. On a connected conformal manifold $(M, \mathcal{C})$ let $g \in \mathcal{C}$. Suppose that there is a nonzero function $u \in \operatorname{ker} P_{g}$ that is either everywhere nonnegative, or everywhere nonpositive. Then there is no nonzero constant function in $\mathcal{R}(Q)$.

Remark A.6. Note the result in the proposition is interesting only if $u$, as described there, has a nontrivial zero locus. Otherwise, if $u$ is strictly positive or strictly negative, then it follows easily from (A.1) that there is a metric $\hat{g} \in \mathcal{C}$ such that $P_{\hat{g}}$ annihilates (all) constant functions and so the constant function 0 is in $\mathcal{R}(Q)$, see Proposition 5.8.

\section{A.5 Final comments}

Although we have focussed on the GJMS operators the results here apply more widely. We could replace the GJMS operators with any conformally covariant and formally self-adjoint operator $\bar{P}_{k}$ (on functions) of the same conformal bidegree and taking the form

$$
\bar{P}_{k}=\delta \bar{S}_{k} d+\bar{Q}_{k}
$$

(cf. (2.7)) with $\bar{Q}_{k}$ nontrivial. Then the $\bar{Q}_{k}$ prescription theory would mirror that above. Alternative conformal powers of the Laplacian, with these properties, are described in [43] (due to M.G. Eastwood and the first author).

Similarly, in certain circumstances the restriction $2 k \notin\{n+2, n+4, \ldots\}$, on even manifolds, can be relaxed. For example, this is the case if the manifold is locally conformally flat or if it is locally conformally Einstein [44]; in both settings there is a class of differential operators which extends the GJMS family to these orders.

\section{Special Remarks}

R.G. acknowledges the support and hospitality of SISSA during 2009 visit when the Appendix material was conceived. A.M. is supported by the project FIRB-Ideas Analysis and Beyond and by the MiUR project Variational methods and nonlinear PDEs. 


\section{References}

[1] Alexakis, S. "On conformally invariant differential operators in odd dimension." Proceedings of the National Academy of Sciences of the United States of America 100, no. 2 (2003): 4409-10.

[2] Alexakis, S. On conformally invariant differential operators. preprint arXiv:math/0608771.

[3] Ammann, B., M. Dahl, and E. Humbert. "Surgery and harmonic spinors." Advances in Mathematics 220, no. 2 (2009): 523-39.

[4] Aubin. T. Some Nonlinear Problems in Riemannian Geometry. Springer Monographs in Mathematics. Berlin: Springer, 1998.

[5] Bär, C. and M. Dahl. "Small eigenvalues of the conformal Laplacian." Geometric and Functional Analysis 13, no. 3 (2003): 483-508.

[6] Baird, P., A. Fardoun, and R. Regbaoui. "Prescribed $Q$-curvature on manifolds of even dimension." Journal of Geometry and Physics 59, no. 2 (2009): 221-33.

[7] Bogomolny, E. and C. Schmit. "Percolation model for nodal domains of chaotic wave functions." Physical Review Letters 88, no. 11 (2002): 114102.

[8] Bourguignon, J.-P. and J.-P. Ezin. "Scalar curvature functions in a conformal class of metrics and conformal transformations." Transactions of the American Mathematical Society 301, no. 2 (1987): 723-36.

[9] Branson. T. "Differential operators canonically associated to a conformal structure." Mathematica Scandinavica 57, no. 2 (1985): 293-345.

[10] Branson. T. P. The Functional Determinant, vi + 103 pp. Lecture Notes Series 4. Seoul: Seoul National University, Research Institute of Mathematics, Global Analysis Research Center, 1993.

[11] Branson, T. P. "Sharp inequalities, the functional determinant, and the complementary series." Transactions of the American Mathematical Society 347, no. 10 (1995): 3671-742.

[12] Branson, T. P., S.-Y. A. Chang, and P. Yang. "Estimates and extremals for zeta function determinants on four-manifolds." Communications in Mathematical Physics 149, no. 2 (1992): 241-62.

[13] Branson, T. P. and A. R. Gover. Origins, Applications and Generalisations of the Q-curvature. American Institute of Mathematics, 2003. http://www.aimath.org/ pastworkshops/confstruct.html.

[14] Branson, T. and A. Rod Gover. "Origins, applications and generalisations of the Q-curvature." Acta Applicandae Mathematicae 102, no. 2-3 (2008): 131-46.

[15] Branson, T. P. and B. Ørsted. "Conformal geometry and global invariants." Differential Geometry and its Applications 1, no. 3 (1991): 279-308.

[16] Branson, T. P. and B. Ørsted. "Explicit functional determinants in four dimensions." Proceedings of the American Mathematical Society 113, no. 3 (1991): 669-82.

[17] Brendle. S. "Convergence of the $Q$-curvature flow on $S^{4}$." Advances in Mathematics 205, no. 1 (2006): 1-32.

[18] Brezin, J. "Harmonic analysis on nilmanifolds." Transactions of the American Mathematical Society 150 (1970): 611-8. 
[19] Buser, P. “Riemannsche Flächen mit Eigenwerten in (0, 1/4)." Commentarii Mathematici Helvetici 52, no. 1 (1977): 25-34.

[20] Canzani, Y., R. Gover, D. Jakobson, and R. Ponge. Nullspaces of conformally invariant operators. Applications to $Q_{k}$-curvature. (2012): preprint arXiv:1206.0517.

[21] Canzani, Y., R. Gover, D. Jakobson. and R. Ponge. “Conformal invariants from nodal sets. II." (2012): preprint.

[22] Chang, S.-Y. A., M. J. Gursky, and P. C. Yang. "A conformally invariant sphere theorem in four dimensions." Publications Mathématiques. Institut de Hautes Études Scientifiques 98 (2003): 105-43.

[23] Chang, S.-Y. A. and P. C. Yang. “Extremal metrics of zeta function determinants on 4-manifolds." Annals of Mathematics (2) 142, no. 1 (1995): 171-212.

[24] Cheng, S. Y. "Eigenfunctions and nodal sets." Commentarii Mathematici Helvetici 51, no. 1 (1976): 43-55.

[25] Cheng, S.-Y. and S.-T. Yau. "Differential equations on Riemannian manifolds and their geometric applications." Communications on Pure and Applied Mathematics 28, no. 3 (1975): 333-54.

[26] Chernov, V. "Framed knots in 3-manifolds and affine self-linking numbers." Journal of Knot Theory and its Ramifications 14, no. 6 (2005): 791-818.

[27] Chernov, V. and Y. Rudyak. "Toward a general theory of linking invariants." Geometry and Topology 9 (2005): 1881-913.

[28] Courant, R. and D. Hilbert. Methods of Mathematical Physics, vol. I, xv+561 pp. New York, NY: Interscience Publishers, Inc., 1953.

[29] Delanoë, P. and F. Robert. "On the local Nirenberg problem for the $Q$-curvatures." Pacific Journal of Mathematics 231, no. 2 (2007): 293-304.

[30] Djadli, Z. and A. Malchiodi. "Existence of conformal metrics with constant $Q$-curvature." Annals of Mathematics. Second Series 186, no. 3 (2008): 813-58.

[31] Donnelly, H. and C. Fefferman. "Nodal sets of eigenfunctions on Riemannian manifolds." Inventiones Mathematicae 93, no. 1 (1988): 161-83.

[32] Dirac, P. A. M. "Wave equations in conformal space." Annals of Mathematics 37, no. 2 (1936): 429-42.

[33] Eastwood, M. G. and J. Slovák. "Semiholonomic Verma modules." Journal of Algebra 197, no. 2 (1997): 424-48.

[34] Fardoun, A. and R. Regbaoui. " $Q$-curvature flows for GJMS operators with nontrivial kernel." Journal of Geometry and Physics 62, no. 12 (2012): 2321-28.

[35] Federer, H. Geometric Measure Theory, 680 pages. Die Grundlehren der mathematischenWissenschaften 153. Berlin: Springer, 1969.

[36] Fefferman, C. and C. R. Graham. Conformal Invariants, 95-116. Élie Cartan et les Mathématiques d'Aujourd'hui, Astérisque, hors série, 1985.

[37] Fefferman, C. and C. R. Graham. "Q-curvature and Poincaré metrics." Mathematical Research Letters 9, no. 2-3 (2002): 139-51.

[38] Fefferman, C. and C. R. Graham. The Ambient Metric, x+113 pp. Annals of Mathematics Studies 178. Princeton, NJ: Princeton University Press, 2012. 
[39] Fischer, A. and V. Moncrief. "The Structure of Quantum Conformal Superspace." In Global Structure and Evolution in General Relativity, edited by S. Gotsakis and G. Gibbons, 111-73. Berlin: Springer, 1996.

[40] Fischer, A. and V. Moncrief. "Hamiltonian reduction of Einstein's equations of general relativity." Nuclear Physics B (Proceedings Supplement) 57 (1997): 142-61.

[41] Folland, G. "Compact Heisenberg manifolds as CR manifolds." The Journal of Geometric Analysis 14, no. 3 (2004): 521-32.

[42] Gordon, C. S. and E. N. Wilson. "The spectrum of the Laplacian on Riemannian Heisenberg manifolds." The Michigan Mathematical Journal 33, no. 2 (1986): 253-71.

[43] Gover, A. R. "Aspects of Parabolic Invariant Theory." The 18th Winter School "Geometry and Physics" (Srní 1998). Rendiconti del Circolo Matematico di Palermo. Serie II. Supplemento No. 59 (1999) 25-47.

[44] Gover, A. R. "Laplacian operators and Q-curvature on conformally Einstein manifolds." Mathematische Annalen 336, no. 2 (2006): 311-34.

[45] Gover, A. R. "O curvature prescription; forbidden functions and the GJMS null space." Proceedings of the American Mathematical Society 138, no. 4 (2010): 1453-9.

[46] Gover, A. R. and K. Hirachi. "Conformally invariant powers of the Laplacian-a complete non-existence theorem." Journal of the American Mathematical Society 17, no. 2 (2004): 389-405.

[47] Gover, A. R. and L. J. Peterson. “Conformally invariant powers of the Laplacian, $Q$-curvature, and tractor calculus." Communications in Mathematical Physics 235, no. 2 (2003): 339-78.

[48] Graham, C. R. "Conformally invariant powers of the Laplacian. II. Nonexistence." Journal of the London Mathematical Society. Second Series 46, no. 3 (1992): 566-76.

[49] Graham, C. R. Talk at the workshop Conformal Structure in Geometry, Analysis, and Physics. Palo Alto: AIM, August 12-16, 2003.

[50] Graham, C. R., R. Jenne, L. J. Mason, and G. A. Sparling. “Conformally invariant powers of the Laplacian, I: existence." The Journal of the London Mathematical Society 46, no. 3 (1992): 557-65.

[51] Graham, C. R. and M. Zworski. "Scattering matrix in conformal geometry." Inventiones Mathematicae 152, no. 1 (2003): 89-118.

[52] Gromov, M. and H. Blaine Lawson. "The classification of simply connected manifolds of positive scalar curvature." Annals of Mathematics. Second Series 111, no. 3 (1980): 423-34.

[53] Guillarmou, C. Private communication.

[54] Guillarmou, C. and F. Naud. "Wave 0-trace and length spectrum on convex co-compact hyperbolic manifolds." Communications in Analysis and Geometry 14, no. 5 (2006): 945-67.

[55] Guillarmou, C. and J. Oing. "Spectral characterization of Poincaré-Einstein manifolds with infinity of positive Yamabe type." International Mathematics Research Notices 2010, no. 9, 1720-1740.

[56] Han, Q. "Singular sets of solutions to elliptic equations." Indiana University Mathematics Journal 43, no. 3 (1994): 983-1002. 
[57] Han, Q., R. Hardt, and F. H. Lin. "Geometric measure of singular sets of elliptic equations." Communications on Pure and Applied Mathematics 51, no. 11-12 (1998): 1425-43.

[58] Hardt, R., M. Hoffmann-Ostenhof, T. Hoffmann-Ostenhof, and N. Nadirashvili. "Critical sets of solutions to elliptic equations." Journal of Differential Geometry 51, no. 2 (1999): 359-73.

[59] Jensen, G. "The scalar curvature of left invariant Riemannian metrics." Indiana University Mathematics Journal 20, no. 12 (1971): 1125-44.

[60] Juhl, A. Families of Conformally Covariant Differential Operators, Q-curvature and Holography, 500 pages. Progress in Mathematics 275. Basel: Birkhäuser, 2009.

[61] Katagiri, M. "On the topology of the moduli space of negative constant scalar curvature metrics on a Haken manifold." Proceedings of the Japan Academy 75(A), no. 7 (1999): 126-8.

[62] Kazdan, J. L. and F. W. Warner. "Curvature functions for compact 2-manifolds." Annals of Mathematics. Second Series 99, no. 1 (1974): 14-47.

[63] Kazdan, J. and F. Warner. "Scalar curvature and conformal deformations of Riemannian structure." Journal of Differential Geometry 10, no. 1 (1975): 113-34.

[64] Kodaira, K. and D. Spencer. "On deformations of complex analytic structures, III. Stability theorems for complex structures." Annals of Mathematics 71, no. 1 (1960): 43-76.

[65] Lohkamp, J. "The space of negative scalar curvature metrics." Inventiones Mathematicae 110, no. 2 (1992): 403-7.

[66] Lohkamp, J. "Discontinuity of geometric expansions." Commentarii Mathematici Helvetici 71, no. 2 (1996): 213-28.

[67] Maier, S. "Generic metrics and connections on Spin- and Spin $^{C}$-manifolds." Communications in Mathematical Physics 188, no. 2 (1997): 407-37.

[68] Malchiodi, A. "Conformal metrics with constant $Q$-curvature." Symmetry, Integrability and Geometry: Methods and Applications 3 (2007): Paper 120, 11 pages.

[69] Malchiodi, A. and M. Struwe. "Q-curvature flow on $S^{4}$." Journal of Differential Geometry 73, no. 1 (2006): 1-44.

[70] Nazarov, F. and M. Sodin. "On the number of nodal domains of random spherical harmonics." American Journal of Mathematics 131, no. 5 (2009): 1337-57.

[71] Ndiaye, C. B. "Constant $Q$-curvature metrics in arbitrary dimension." Journal of Functional Analysis 251, no. 1 (2007): 1-58.

[72] Okikiolu, K. "Critical metrics for the determinant of the Laplacian in odd dimensions." Annals of Mathematics. Second Series 153, no. 2 (2001): 471-531.

[73] Osgood, B., R. Phillips, and P. Sarnak. "Extremals of determinants of Laplacians." Journal of Functional Analysis 80, no. 1 (1988): 148-211.

[74] Paneitz, S. "A quartic conformally covariant differential operator for arbitrary pseudoRiemannian manifolds." (1983): preprint. Reproduced as: SIGMA 4 (2008), 036, 3 pages.

[75] Parker, T. and S. Rosenberg. "Invariants of conformal Laplacians." Journal of Differential Geometry 25, no. 2 (1987): 199-222.

[76] Pleijel, A. "Remarks on Courant's nodal line theorem." Communications on Pure and Applied Mathematics 9, no. 3 (1956): 543-50. 
[77] Rosenberg, J. "Manifolds of Positive Scalar Curvature: A Progress Report." Surveys in Differential Geometry, vol. XI, 259-94. Surveys in Differential Geometry 11. Somerville, MA: International Press, 2007.

[78] Sogge, C. and S. Zelditch. "Lower bounds on the Hausdorff measure of nodal sets." Mathematical Research Letters 18, no. 1 (2011): 25-37.

[79] Toth, J. and S. Zelditch. "Counting nodal lines which touch the boundary of an analytic domain." Journal of Differential Geometry 81, no. 3 (2009): 649-86.

[80] Wünsch, V. “On conformally invariant differential operators." Mathematische Nachrichten 129, no. 1 (1986): 269-81. 